\title{
Enhancer of polycomb maintains germline activity and genome integrity in Drosophila testis
}

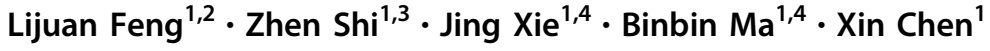

Received: 28 June 2017 / Revised: 20 November 2017 / Accepted: 11 December 2017 / Published online: 23 January 2018

(c) ADMC Associazione Differenziamento e Morte Cellulare 2018

\begin{abstract}
Tissue homeostasis depends on the ability of tissue-specific adult stem cells to maintain a balance between proliferation and differentiation, as well as ensure DNA damage repair. Here, we use the Drosophila male germline stem cell system to study how a chromatin factor, enhancer of polycomb $[\mathrm{E}(\mathrm{Pc})]$, regulates the proliferation-to-differentiation (mitosis-to-meiosis) transition and DNA damage repair. We identified two critical targets of $\mathrm{E}(\mathrm{Pc})$. First, $\mathrm{E}(\mathrm{Pc})$ represses $C y c B$ transcription, likely through modulating $\mathrm{H} 4$ acetylation. Second, $\mathrm{E}(\mathrm{Pc})$ is required for accumulation of an important germline differentiation factor, Bag-of-marbles $(\mathrm{Bam})$, through post-transcriptional regulation. When $\mathrm{E}(\mathrm{Pc})$ is downregulated, increased $\mathrm{CycB}$ and decreased Bam are both responsible for defective mitosis-to-meiosis transition in the germline. Moreover, DNA double-strand breaks (DSBs) accumulate upon germline inactivation of $\mathrm{E}(\mathrm{Pc})$ under both physiological condition and recovery from heat shock-induced endonuclease expression. Failure of robust DSB repair likely leads to germ cell loss. Finally, compromising the activity of Tip60, a histone acetyltransferase, leads to germline defects similar to $E(P c)$ loss-of-function, suggesting that $\mathrm{E}(\mathrm{Pc})$ acts cooperatively with Tip60. Together, our data demonstrate that $\mathrm{E}(\mathrm{Pc})$ has pleiotropic roles in maintaining male germline activity and genome integrity. Our findings will help elucidate the in vivo molecular mechanisms of $\mathrm{E}(\mathrm{Pc})$.
\end{abstract}

Edited by E Baehrecke

Zhen Shi, Jing Xie, and Binbin Ma contributed equally to this work.

Electronic supplementary material The online version of this article (https://doi.org/10.1038/s41418-017-0056-5) contains supplementary material, which is available to authorized users.

Xin Chen

xchen32@jhu.edu

1 Department of Biology, The Johns Hopkins University, Baltimore, MD 21218, USA

2 Laboratory of Chromatin Biology and Epigenetics, The Rockefeller University, New York, NY 10065, USA

3 Geometry Technologies LLC, 6-302, 289 Bisheng Lane, Zhangjiang, Shanghai 201204, China

4 Clinical Research Center for Stem Cell Therapy and Institute for Regenerative Medicine, Shanghai East Hospital; School of Life Sciences and Technology, Tongji University, Shanghai 200092, China

\section{Introduction}

Stem cell proliferation and differentiation require precise regulation to achieve balanced tissue homeostasis. Drosophila spermatogenesis provides a great model system to study the molecular mechanisms of stem cell proliferation versus differentiation decision under physiological conditions [1]. Spermatogenesis initiates from the asymmetric cell division of germline stem cells (GSCs), generating a self-renewed GSC and a gonialblast, which undergoes fourround mitosis as the transit-amplifying spermatogonia. After mitosis, 16 fusome-connected germ cells enter meiosis with a prolonged G2-phase as spermatocytes, followed by two rounds of meiotic divisions. The transition from mitotic spermatogonia to meiotic spermatocytes requires a differentiation factor called Bag-of-marbles (Bam), the loss of which leads to continuously dividing spermatogonia [2-7].

Chromatin regulators that change the epigenetic status of target genes without altering their primary DNA sequences play critical roles in GSC lineages [8]. High-throughput mRNA sequencing (RNA-seq) revealed that chromatin remodeling factors and histone-modifying enzymes are 
enriched in mitotic germline-enriched bam mutant testes compared with meiotic germline-containing wild-type testes [9], suggesting their potential roles in regulating the mitosisto-meiosis transition.

During the mitotic stage, approximately $20 \%$ of spermatogonial cysts, mainly at the 4- to 16-cell stage, undergo spontaneous cell death, which could serve as a quality control [10-12] to maintain germline genome integrity by eliminating cells with unrepaired DNA damage [13]. During DNA repair, chromatin modification is essential to allow repair proteins to access the damage sites. For example, phosphorylation of histone variant $\mathrm{H} 2 \mathrm{AX}$ at DNA double-strand break (DSB) sites facilitates recognition by repair proteins [14].

Among known chromatin regulators acting in DNA damage response, Tip60 contributes to DNA repair through its ATPase activity [15]. Tip60 has dual roles as both ATPdependent chromatin remodeling enzyme and histone acetyltransferase (HAT) [16]. Tip60 can acetylate lysine residues of $\mathrm{H} 4, \mathrm{H} 2 \mathrm{~A}$, and transcription factors, to activate gene expression by decondensing chromatin and allowing transcriptional machinery to access DNA [17].

$\mathrm{E}(\mathrm{Pc})$ has been characterized as one component of the Drosophila Tip60 (dTip60) complex biochemically [18]. Genetic assays identified $E(P c)$ mutant as an enhancer of Polycomb mutant and a suppressor of position-effect variegation $[19,20]$. Here we report that $\mathrm{E}(\mathrm{Pc})$ promotes mitosis-to-meiosis transition through repressing $C y c B$ transcription and promoting Bam protein accumulation in the Drosophila male germline. Moreover, inactivating $\mathrm{E}(\mathrm{Pc})$ in germ cells leads to increased DSBs and cell death. $\mathrm{E}(\mathrm{Pc})$ is required for the HAT activity of dTip60. Compromising HAT activity of dTip60 phenocopies $E(P c)$ loss-of-function defects. Together, our findings reveal heretofore poorly understood in vivo pleiotropic roles of $\mathrm{E}(\mathrm{Pc})$.

\section{Results}

\section{$E(P c)$ is required cell autonomously for germline survival and differentiation}

$E(P c)$ is highly expressed in Drosophila adult testes with enrichment in bam mutant compared with wild-type testes, based on RNA-seq analysis (Supplementary Fig. 1a) [9]. A GFP (Green Fluorescent Protein)-tagged genomic-rescuing $E(P c)$ transgene [21] showed nuclear localization of $\mathrm{E}$ (Pc)-GFP fusion protein in GSCs, spermatogonia, and spermatocytes (Supplementary Fig. 1b, c), consistent with previous findings that $\mathrm{E}(\mathrm{Pc})$ encodes a chromatin factor and binds to multiple polytene chromosomal sites [19, 20].

To explore $\mathrm{E}(\mathrm{Pc})$ 's functions in germ cells, a short hairpin RNA (shRNA) targeting its coding sequences was driven by an early-stage germline driver nanos-Gal4 (nosGal4) for knockdown (KD) experiments. In nos $>E(P c)$ shRNA testes, the $\mathrm{E}(\mathrm{Pc})$-GFP signal was diminished in germ cells from GSCs to spermatogonia, but still detectable in somatic gonadal cells (Supplementary Fig. 1d, e), suggesting a germline-specific KD.

To investigate germline defects specifically in adult testes, the temperature-sensitive Gal80 controlled by the tubulin promoter (tub-Gal80 ${ }^{t s}$ ) was applied to temporally express $E(P c)$ shRNA. At the permissive temperature, both tub-Gal80 ${ }^{t s}$, nos-Gal4 (Ctrl), and tub-Gal80 ${ }^{t s}$, nos $>E(P c)$ shRNA $[E(P c) \mathrm{KD}]$ testes had germ cells at all stages, including GSCs, spermatogonia, and spermatocytes (Figs. 1a, b). However, 5 days after shifting to the restrictive temperature, cysts with $>16$ germ cells were found in $55 \%$ of $E(P c) \mathrm{KD}(N=20)$ (cyan outline, Fig. 1d) but not in $\mathrm{Ctrl}$ $(N=28$, Fig. 1c) testes. Such phenotype became more severe in $39 \%$ of $E(P c)$ KD testes $(N=23) 10$ days after temperature shift (Fig. 1f, average spermatogonial tumors/ testis was 5 at day 10 vs. 2.4 at day 5).

Furthermore, when using lysotracker to detect germ cell death $[10,11]$, more than three lysotracker-positive cysts could be found in $\sim 30 \%$ of $E(P c)$ KD testes (Fig. 1f) but in only $5 \%$ of $C t r l$ testes $(N=18$, Fig. 1e). This increased cell death might explain germ cell loss in $E(P c) \mathrm{KD}$ testes (Figs. 1g, h). A time-course quantification (Fig. 1i) revealed that GSC number between $\operatorname{Ctrl}(N=18,10.22 \pm 1.77$, mean $\pm \mathrm{SD})$ and $E(P c) \mathrm{KD}(N=45,11.04 \pm 1.95)$ testes was comparable at the permissive temperature. But a significant GSC decrease $\left(P<10^{-4}\right)$ was found in $E(P c) \mathrm{KD}$ $(N=20,7.95 \pm 2.04)$ compared with $\operatorname{Ctrl}(N=18,11.72 \pm$ $2.37)$ testes 5 days after temperature shift. At 10 days after temperature shift, GSC number in $E(P c) \mathrm{KD}(N=23,7.09$ $\pm 4.18)$ became more comparable to $\operatorname{Ctrl}(N=18,9.67 \pm$ $1.97)$ testes. However, some of these $E(P c)$ KD testes were devoid of late-stage germ cells (Fig. 1h). Together, these results indicate that $\mathrm{E}(\mathrm{Pc})$ is required cell autonomously for germ cell survival, the mitosis-to-meiosis transition, and GSC maintenance.

Next, we generated $E(P c)$ mutant clones using the Flippase/Flippase Recognition Target system with a strong loss-of-function $E(P c)^{w 3}$ allele [22]. At 2 days after clonal induction (ACI), 65\% of testes $(N=43)$ had at least one $E$ (Pc) GSC clone, comparable to $\mathrm{Ctrl}$ testes with wild-type GSC clones (77\%, $N=66$; Fig. 1p), suggesting similar clonal induction efficiency. However, the percentage of testes with $E(P c)$ GSC clones declined much faster than that of $\mathrm{Ctrl}$ testes (Fig. 1p). No testis with $E(P c)$ GSC clone could be found 8 days ACI (Fig. 1p). These data confirmed that $\mathrm{E}(\mathrm{Pc})$ is required cell autonomously for $\mathrm{GSC}$ maintenance.

Although no obvious difference was found for the percentage of testes with spermatogonial clones 4 days ACI, 

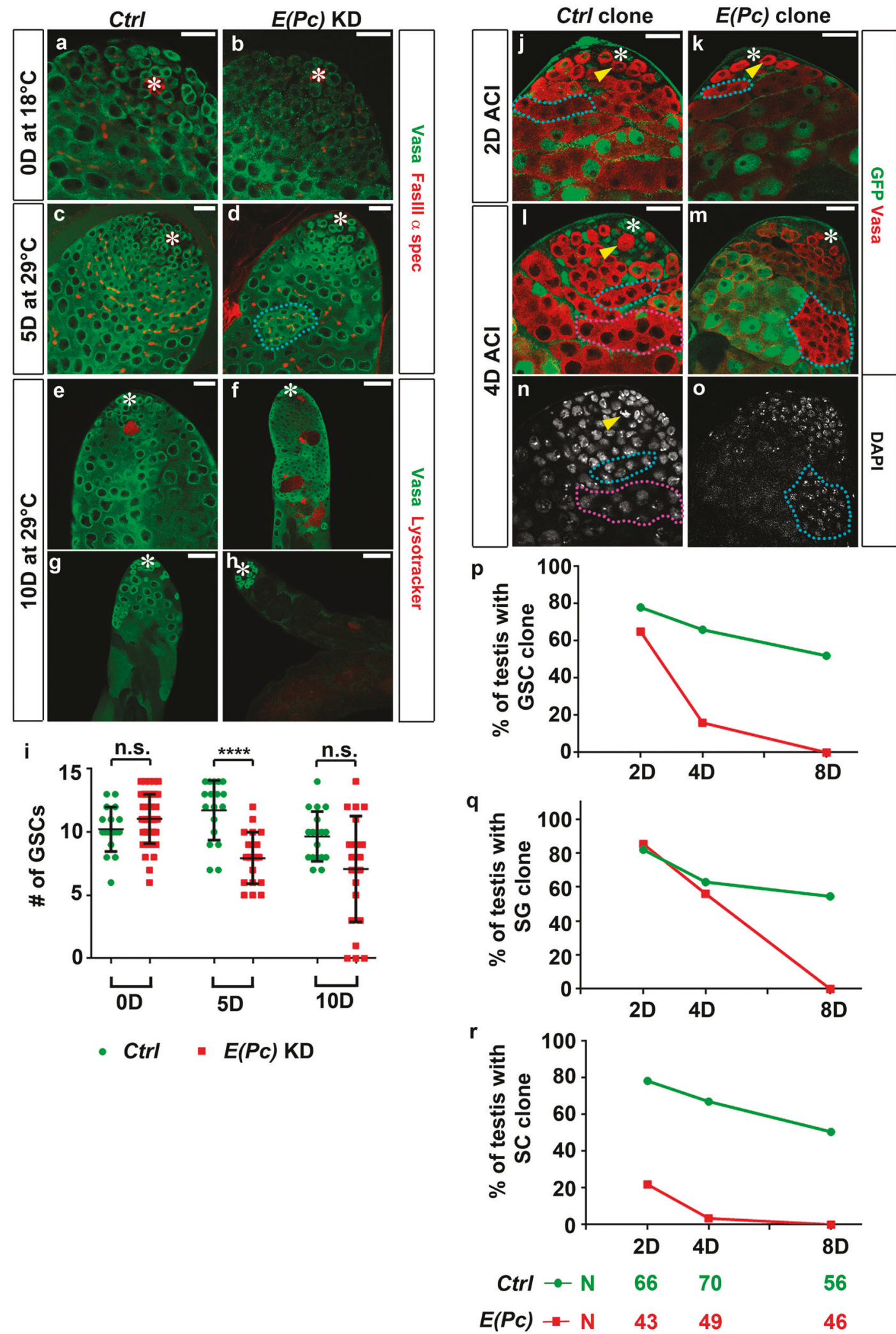

$17.5 \%$ of $E(P c)$ spermatogonial clones $(N=97)$ had $>16$ germ cells (cyan outline, Figs. 1m, o), consistent with the spermatogonial tumor found in $E(P c) \mathrm{KD}$ testes (cyan outline, Fig. 1d). Moreover, the $E(P c)$ spermatogonial clone was undetectable 8 days ACI (Fig. 1q), likely attributed to decreased GSCs and increased spermatogonial cell death. 
Fig. $1 \mathrm{E}(\mathrm{Pc})$ is required cell autonomously for GSC maintenance and germ cell differentiation. tub-Gal80 $0^{t s}$, nos-Gal4 (Ctrl), and tub$G_{a l 80^{t s}}$, nos $>E(P c)$ shRNA $[E(P c) \mathrm{KD}]$ testes at a, b permissive temperature $\left(18^{\circ} \mathrm{C}\right)$ and $\mathbf{c}-\mathbf{h}$ restrictive temperature $\left(29^{\circ} \mathrm{C}\right)$, immunostained with Vasa to label germ cells, FasIII for hub (asterisk) and $\alpha$ spectrin for spectrosome and fusome. i Quantification of GSCs number (average $\pm \mathrm{SD}$ ). Mann-Whitney $U$-test. n.s. nonsignificant, $* * * * P<$ 0.0001 . j-o GFP was used to identify $C t r l$ and $E(P c)$ mutant clones. jk 2D after clonal induction (ACI), GFP-negative Ctrl GSC clone (yellow arrowhead in $\mathbf{j}$ ), and $E(P c)$ mutant GSC clone (yellow arrowhead in k); GFP-negative Ctrl spermatogonial (SG) clone (cyan outline in $\mathbf{j}$ ) and $E(P c)$ mutant SG clone (cyan outline in $\mathbf{k}$ ). $\mathbf{l}, \mathbf{n} 4 \mathrm{D}$ ACI, Ctrl GSC clone (yellow arrowhead), SG clone (cyan outline), and spermatocyte (SC) clone (magenta outline). m, o 4D ACI, $E(P c)$ mutant SG clone with bright DAPI intensity o has $>16$ germ cells within one cyst (cyan outline). No GSC clone or SC clone could be found for testes shown. $\mathbf{p}-\mathbf{r}$ Percentage of testes with GSC clone $\mathbf{p}, \mathrm{SG}$ q, SC $\mathbf{r}$ clones. $N$ at the bottom represents the number of total testes from three independent clonal induction experiments. For each experiment, number of testes with at least one GFP-negative clone/ total testes number was calculated and presented as $\%$ of testes with the corresponding type of clone. Asterisk: hub. Scale bar for $\mathrm{f}, \mathrm{g}, \mathrm{h}$ : 50 $\mu \mathrm{m}$. Scale bar for other panels: $20 \mu \mathrm{m}$.

Furthermore, at any time point ACI, very few $E(P c)$ spermatocyte clones was found (Figs. 1k, m, r), consistent with previous KD results (Figs. 1d, f).

Furthermore, clonal induction using another loss-offunction $E(P c)^{l}$ allele [19] showed similar germline phenotypes (Supplementary Fig. 2). Together, both germline $\mathrm{KD}$ and clonal analyses demonstrate that $\mathrm{E}(\mathrm{Pc})$ acts cell autonomously to maintain germline including GSCs and promote the mitosis-to-meiosis transition.

\section{$\mathrm{E}(\mathrm{Pc})$ represses $\mathrm{CycB}$ transcription to restrict spermatogonial overproliferation}

To understand the molecular mechanisms underlying spermatogonial tumor phenotype, we examined a critical cell cycle regulator $\mathrm{CycB}$ by immunostaining. In $\mathrm{Ctrl}$ testes, $\mathrm{CycB}$ was detected in the mitotic germ cells (yellow solid line, Figs. 2a-c and Supplementary Fig. 3a-c, 3d-f) but downregulated upon completion of mitosis and became undetectable in meiotic spermatocytes (white solid line, Fig. 2c). CycB was detectable again at the G2/M transition of meiosis I (magenta solid line, Supplementary Fig. 3a-c), as reported previously [23, 24]. By contrast, in $66 \%$ of $E$ $(P c)$ KD testes $(N=21)$, CycB-positive cells were separated from the apical tip (cyan outline, Figs. 2d-f) with bright 4,6diamidino-2-phenylindole (DAPI) staining and condensed nuclear morphology, suggesting their identity as overproliferating spermatogonia. Noticeably, the $\mathrm{CycB}$ signal in early-stage germ cells did not change dramatically (Figs. 2f' vs. c'), suggesting that misexpression of $\mathrm{CycB}$ upon $E(P c)$ $\mathrm{KD}$ is stage specific.
In addition, nucleoside analog 5-ethynyl-2'-deoxyuridine (EdU) incorporation assay identified cysts with 32 EdUpositive spermatogonia in $21.9 \%$ of $E(P c)$ KD testes $(N=$ 32). However, only $\leqslant 16$ EdU-positive spermatogonia within one cyst could be detected in Ctrl testes, confirming spermatogonial overproliferation in $E(P c) \mathrm{KD}$ testes. Finally, most $E(P c)$ spermatogonial clones (cyan outline, Figs. 2g-h, 92\%, $N=26$ ) showed increased CycB signal $(N=26$, Fig. 2i).

Next, we halved $\mathrm{CycB}$ level using a null $C y c B^{2}$ allele [25]. We found decreased percentage of testes with spermatogonial tumors from $43 \%$ in $E(P c)$ KD testes $(N=28)$ to $5 \%$ in $C y c B^{2} /+, E(P c)$ KD testes $(N=40)$ (Fig. 2j, $P<10^{-4}$ ). These results suggest that enhanced $\mathrm{CycB}$ levels upon inactivating $E(P c)$ does, at least partially, contribute to the spermatogonial overproliferation phenotype.

Then, $C y c B$ transcript level was measured using quantitative reverse transcriptase-PCR (RT-PCR). An approximate twofold increase of $C y c B$ mRNA was detected in $E$ (Pc) KD testes (tub-Gal80 ${ }^{t s}$, nos $>E(P c)$ shRNA) compared with Ctrl testes (tub-Gal80 ${ }^{t s}$, nos-Gal4) (Fig. 2k, $P=0.02$ ), suggesting that $\mathrm{E}(\mathrm{Pc})$ likely regulates $C y c B$ transcription. Next, chromatin immunoprecipitation (ChIP)-seq experiments were performed to investigate whether $\mathrm{E}(\mathrm{Pc})$ binds to the $C y c B$ genomic region in Drosophila $\mathrm{S} 2$ cells, which has high $\mathrm{E}(\mathrm{Pc})$ expression based on ModENCODE data. A ChIP-grade GFP antibody [21] was used to pull down E (Pc)-GFP-bound DNA followed by high-throughput sequencing, which identified $5629 \mathrm{E}(\mathrm{Pc})$-bound genes. Enrichment of $\mathrm{E}(\mathrm{Pc})$ was detected within a 600-bp region upstream of transcription start sites (TSSs) (Fig. 2l), confirming $\mathrm{E}(\mathrm{Pc})$ as a chromatin factor [19-21, 26]. Based on ChIP-seq result, enrichment of $\mathrm{E}(\mathrm{Pc})$ was near the TSS of $C y c B$ locus (Fig. 2m). Together, E(Pc) likely downregulates $C y c B$ transcription in spermatogonia for their mitosis-tomeiosis transition. Meanwhile, it is very likely that the pleiotropic $E(P c)$ phenotypes reflect its regulation of many targets including $\mathrm{CycB}$.

\section{$E(P c)$ acts with dTip60 to maintain $\mathrm{H} 4$ acetylation levels in the germline}

Previous biochemical studies have shown that $\mathrm{E}(\mathrm{Pc})$ is a component of the dTip60 HAT complex [18]. The dTip60 has been shown to acetylate both $\mathrm{H} 4$ and $\mathrm{H} 2 \mathrm{~A}$, an activity conserved from yeast [27] to human [15]. Indeed, expression of a dominant-negative HAT-deficient $d T i p 60^{E 431 Q}$ form [28] led to excess spermatogonia (cyan outline, Fig. 3b) and severe germ cell loss (red solid line, Fig. 3c) in $33 \%$ and $17 \%$ of $n o s>d T i p 60^{E 431 Q}$ testes $(N=30)$, respectively. These defects recapitulated $E(P c)$ loss-offunction phenotypes but were not fully penetrant, since 
Fig. $2 \mathrm{E}(\mathrm{Pc})$ in germ cells negatively regulates $C y c B$ transcription. tub-Gal80 ${ }^{t s}$, nosGal4 (Ctrl) testes a-c and tub$G a l 80^{t s}$, nos $>E(P c)$ shRNA [E $(P c) \mathrm{KD}]$ testes $\mathbf{d}-\mathbf{f}$ immunostained with $\mathrm{CycB}$, Vasa and DAPI. c', f' Higher magnification of $\mathrm{CycB}$ staining at the apical tip. g-i GFPnegative $E(P c)$ mutant (cyan outline) clones have $\mathrm{CycB}$ expression. $\mathbf{j}$ Quantification of germline tumor phenotype at different genetic backgrounds. $* * * * P<0.0001$, Chi-square test. k Quantitative RT-PCR of $C y c B$ mRNA in tub-Gal80 ${ }^{t s}$, nos-Gal4 (Ctrl) and tub-Gal80 ${ }^{\text {ts }}$, $n o s>E(P c)$ shRNA $[E(P c) \mathrm{KD}]$ testes. Mean \pm SD (standard deviation) based on three independent biological replicates. Transcript level in $C t r l$ testes set to 1 . Student's $t$ test. $P=0.02$. I ChIP-seq was performed with GFP antibody in S2 cells transfected with ActinGal4 and UAS-E(Pc) $c D N A-$ $G F P$ plasmids. Two independent ChIP experiments were performed. Average E(Pc) signal profile of 5629 target genes over a $-1-\mathrm{kb}$ to $+4-\mathrm{kb}$ region with respect to the TSSs. m A genome browser snapshot of the $C y c B$ gene region. Compared with input, enriched $\mathrm{E}(\mathrm{Pc})$ at genomic region of $C y c B$. Peaks are indicated by magenta lines. The black lines indicate the average read density at chromosome $2 \mathrm{R}$. Two independent replicates are shown here. Asterisk: hub. Scale bar: $20 \mu \mathrm{m}$.
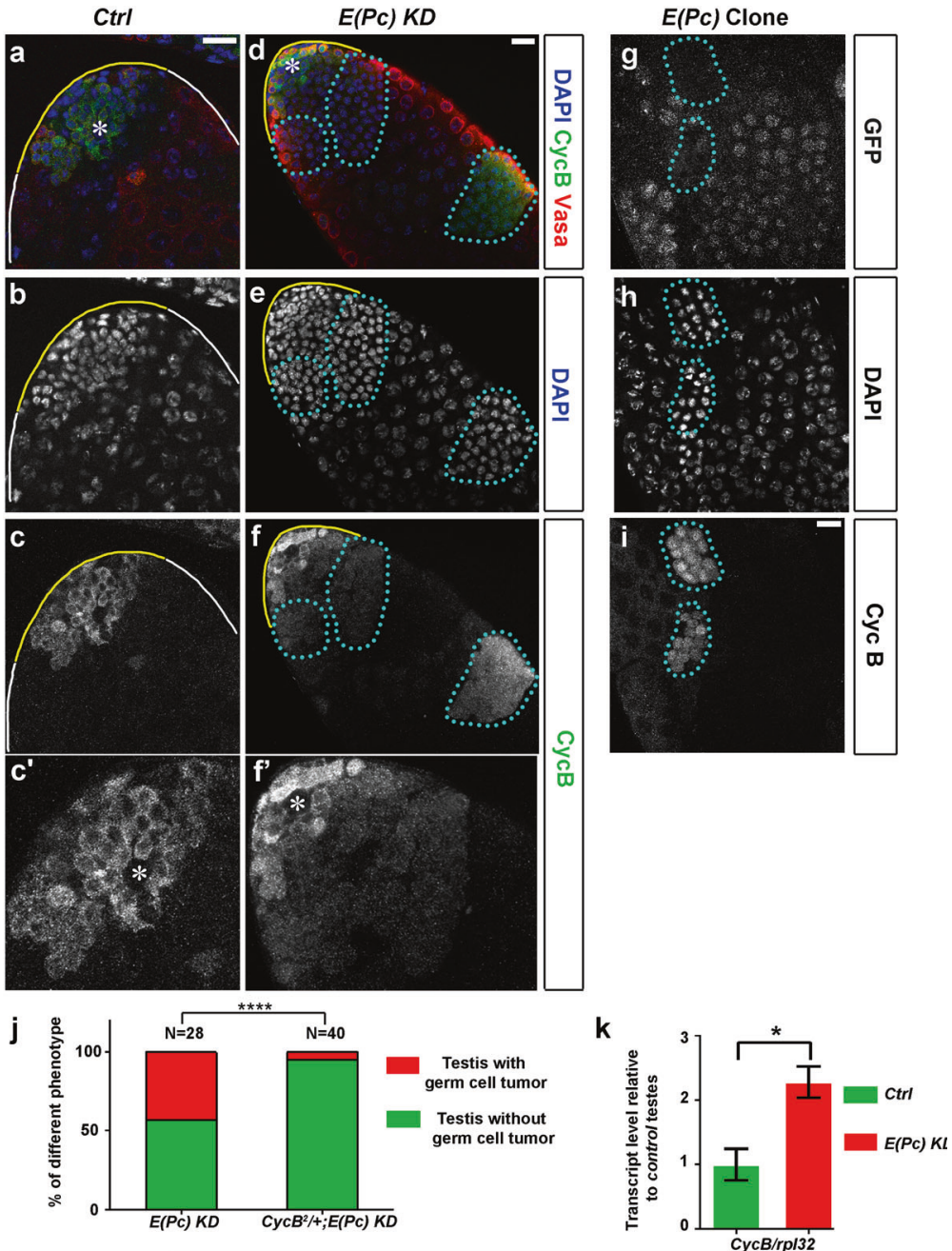

I

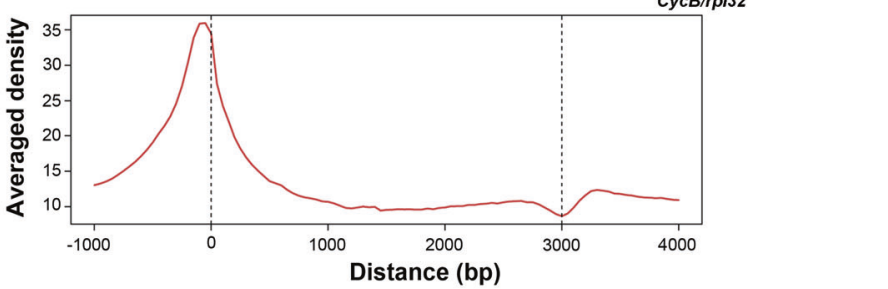

m
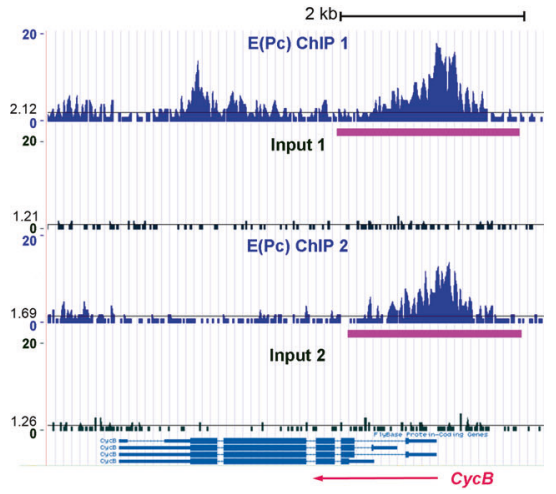

$\sim 50 \%$ of nos $>d$ Tip $60^{E 431 Q}$ testes showed apparently normal morphology (Fig. 3a). However, both germline defects were significantly enhanced upon compromising $\mathrm{E}(\mathrm{Pc})$ with the strong loss-of-function $E(P c)^{l}$ allele [19, 29] (Fig. 3d). Together, these data suggest that $\mathrm{E}(\mathrm{Pc})$ acts cooperatively with dTip60, whose HAT activity is required in germ cells. 
Fig. $3 \mathrm{E}(\mathrm{Pc})$ cooperates with histone acetyltransferase dTip60 to regulate germ cell differentiation through $\mathrm{H} 4$ acetylation. a-c nos $>$ dTip60 ${ }^{E 431 Q}$ testes stained with DAPI, germ cell marker Vasa, hub marker FasIII and fusome marker $\alpha$-spectrin. Normal morphology a, spermatogonial overproliferation (cyan outline in b), and germ cell loss (red solid line in c) phenotypes were detected with different penetrance. d Quantification of germ cell phenotype.

Heterozygous $E(P c)^{l} /+$

enhances germ cell phenotype in nos $>d$ Tip $60^{E 431 Q}$ testes. $* * * P$ $<0.005$. Chi-square test. $\mathbf{e}-\mathbf{p}$ Apical tip of testes immunostained with GFP $\mathbf{f}, \mathbf{j}, \mathbf{n}$ to identify GFP-negative $E(P c)$ mutant clones and DAPI $\mathbf{g}, \mathbf{k}, \mathbf{o}$. q Quantification of intensity of $\mathrm{H} 4$ tetra-acetylation (ace) and H4 immunostaining signals between GFP-positive control cells and GFP-negative $E(P c)$ mutant clones. $\mathrm{H} 4$ ace $\mathrm{atrl}^{\mathrm{ctr}} / \mathrm{H} 4$ ace $^{\text {clone }}=1.66 \pm 0.60 . \mathrm{H} 4^{\text {ctrl } /}$ $\mathrm{H} 4^{\text {clone }}=1.00 \pm 0.17($ mean \pm SD). Two-tailed $t$-test. $* * * * P<$ 0.0001. Asterisk: hub. Scale bar: $20 \mu \mathrm{m}$.
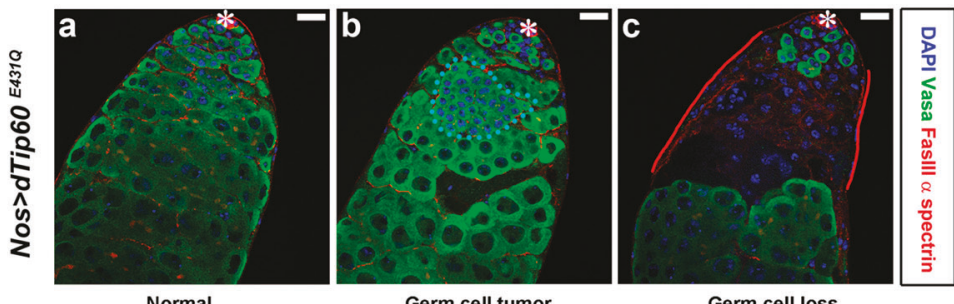
d

Germ cell tumor Germ cell loss
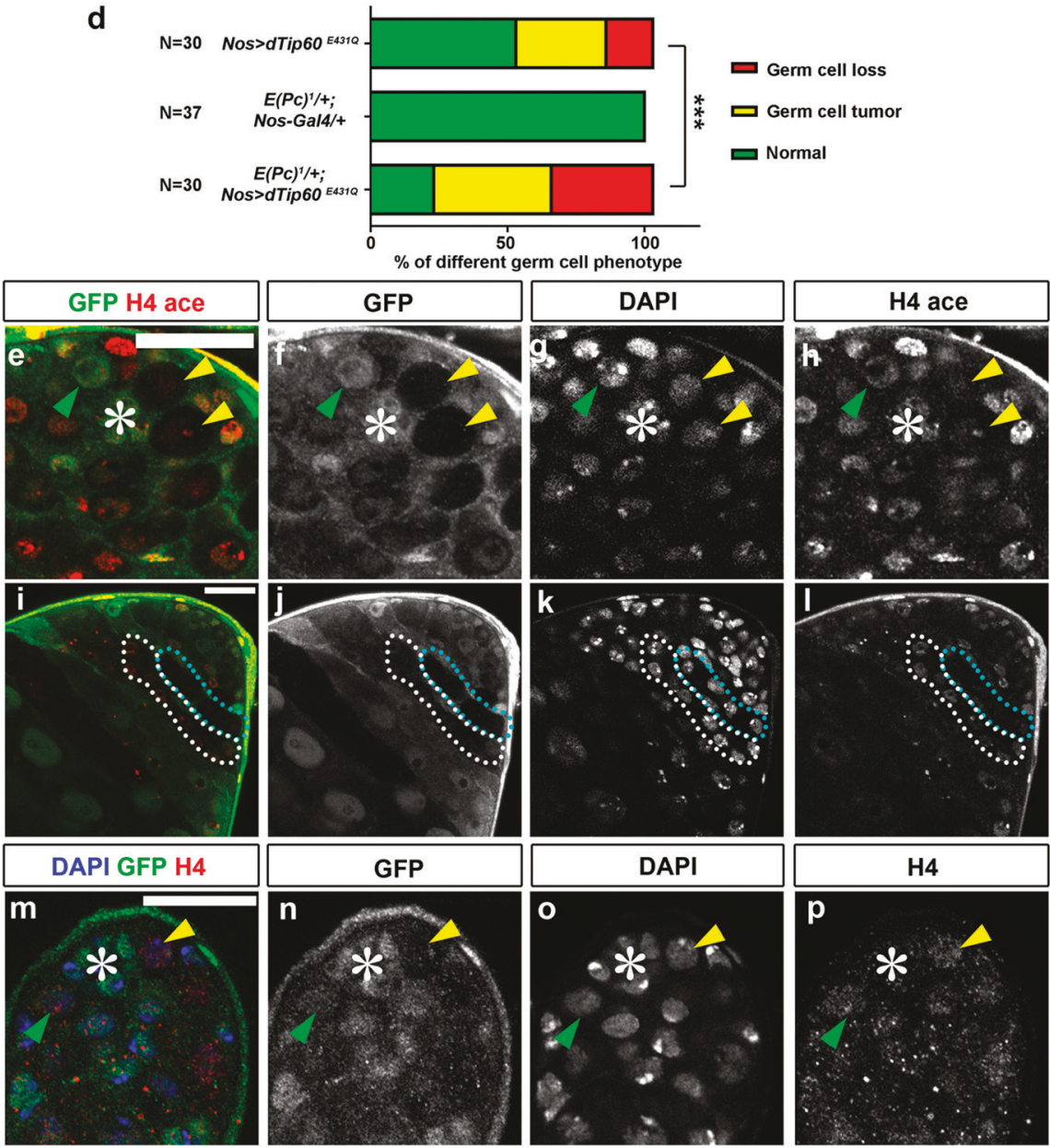

q
Intensity ratio: Heterozygous cells vs. $E(P c)$ clone

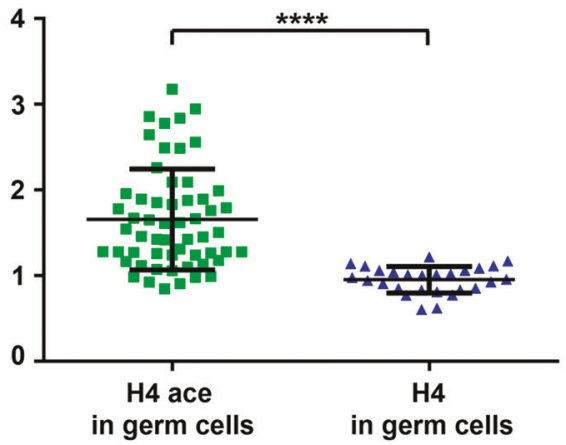

Consistently, tetra-acetylated lysines 5, 8, 12, and 16 of H4 had reduced levels in $E(P c)$ GSC clones $(N=24$, Figs. $3 \mathrm{e}-\mathrm{h})$ and spermatogonial clones $(N=33$, Figs. $3 \mathrm{i}-1)$.
Nevertheless, reduced $\mathrm{H} 4$ tetra-acetylation in $E\left(P_{c}\right)$ mutant germ cells did not result from the change of the overall $\mathrm{H} 4$ level $(N=27$; compare the GSCs indicated by yellow $v s$. 
green arrowheads in Figs. $3 m-p, q)$. Together, these data suggest that downregulation of $\mathrm{E}(\mathrm{Pc})$ compromised the HAT activity of dTip60. Furthermore, ChIP-qPCR experiments using anti-tetra-acetylated $\mathrm{H} 4$ in wild-type testes showed its enrichment near the TSS of $C y c B$ locus (Supplementary Fig. 4), suggesting $\mathrm{E}(\mathrm{Pc})$ might regulate $C y c B$ transcription through $\mathrm{H} 4$ acetylation.

Finally, neither an active $\mathrm{H} 3 \mathrm{~K} 4 \mathrm{me} 3$ mark [30] $(N=29$, Supplementary Fig. 5a-c, g) nor a repressive H3K27me3 mark [31] $(N=33$, Supplementary Fig. 5d-f, g) showed detectable difference between $E(P c)$ and $C t r l$ GSC clones, indicating the specificity of changed $\mathrm{H} 4$ acetylation in $E(P c)$ mutant cells. Interestingly, depletion of Epl1, the yeast homolog of $\mathrm{E}(\mathrm{Pc})$, also leads to global loss of $\mathrm{H} 4$ acetylation [27]. Therefore, the role of $\mathrm{E}(\mathrm{Pc})$ in maintaining $\mathrm{H} 4$ acetylation levels is likely conserved.

\section{$E(P c)$ regulates Bam protein accumulation for the mitosis-to-meiosis transition}

As $E(P c)$ loss-of-function phenotypes (Figs. 1d, m, Supplementary Fig. 2d) resemble the bam mutant phenotype $[2-5,7]$, we next examined Bam expression in $\mathrm{E}(\mathrm{Pc}) \mathrm{KD}$ testes. Cytoplasmic Bam signal was detected in 4-16 cell spermatogonia close to the apical tip, but not much farther away in meiotic spermatocytes in wild-type $(N=18$, Figs. 4a-d) and mcherry KD control $(N=22$, Supplementary Fig. 3g-i) testes, as reported previously [2-4, 6, 7]. In $E$ $(P c)$ KD testes $(N=31)$, germ cells located at the apical tip with normal morphology (Figs. 4e-h) still had detectable Bam (yellow solid line in Fig. 4g'; red signal in Fig. 4h). However, excess germ cells located away from the tip with condensed nuclei (cyan outline, Fig. 4e) and small sizes (cyan outline, Fig. 4f) had reduced Bam in $48 \%$ of $E(P c)$ KD testes $(N=31$, cyan outline, Fig. $4 \mathrm{~g})$. Spermatogonial tumors (Fig. 1d) with reduced Bam were found in $\sim 55 \% \mathrm{E}$ $(P c) \mathrm{KD}$ testes. Consistently, Bam was greatly reduced in overproliferative $E(P c)$ mutant spermatogonial clones $(N=$ 21, cyan outline, Figs. 4i-1). These data suggest that the regulation of $\mathrm{E}(\mathrm{Pc})$ on Bam could be stage specific, because Bam level seemed to normal in early-stage germline (Figs. 4c'vs. g' or k').

Reduced Bam protein could cause spermatogonial overproliferation in both $E(P c) \mathrm{KD}$ (Fig. 1d) and $E(P c)$ mutant clones (Fig. 1m, Supplementary Fig. 2d). To test this, increased Bam expression using a bam-HA transgene [6] at the $E(P c)$ loss-of-function background resulted in complete suppression of the spermatogonial tumor phenotype $(N=37$, Fig. $4 \mathrm{~m})$. This suggests that Bam is an essential downstream factor of $\mathrm{E}(\mathrm{Pc})$ in restricting spermatogonial overproliferation. However, bam mRNA in $E$ $(P C) \mathrm{KD}$ testes was unchanged (Fig. 4n) and no enrichment of $\mathrm{E}(\mathrm{Pc})$ at bam genomic locus could be identified (Fig. 4o), indicating that $\mathrm{E}(\mathrm{Pc})$ regulates bam expression posttranscriptionally.

\section{$E(P c)$ regulates germ cell survival independent of either JAK-STAT or BMP pathway}

Although reducing $C y c B$ level (Fig. 2j) or overexpressing bam (Fig. 4m) could suppress the spermatogonial overproliferation phenotype in $E(P c) \mathrm{KD}$ testes, germ cell loss was still detectable in all $C y c B^{2} /+$, nos $>E(P c) \operatorname{sh} R N A(N$ $=23)$ and bam-HA, nos $>E(P c) \operatorname{sh} N A(N=21)$ testes, suggesting that $\mathrm{E}(\mathrm{Pc})$ regulates germline survival using different mechanism(s). Germ cell loss could be caused by GSC self-renewal defect, impaired GSC division and/or increased cell death.

To examine whether $\mathrm{E}(\mathrm{Pc})$ is required for GSC selfrenewal, several GSC maintenance factors were compared between $E(P c)$ mutant and $C t r l$ GSCs, including the Janus kinase-signal transducer and activator of transcription (JAK-STAT) [32-35] and bone morphogenetic protein (BMP) [5, 36] signaling pathway components, as well as the adherens junctions at the hub cell-GSC interface [34, 37]. We found no evidence on changed expression or localization of STAT92E, phosphor-Mad (pMad), and Drosophila E-cadherin (DE-Cad) between $E(P c)$ and Ctrl GSCs (compare the GSCs indicated by yellow vs. green arrowheads in Fig. 5).

Next, to examine whether $E(P c)$ mutant GSCs have impaired division rate, EdU incorporation assay and phosphor-histone $\mathrm{H} 3$ ( $\mathrm{pH} 3$ ) staining were performed to measure the percentage of S-phase and mitotic GSCs, respectively. The percentages of both EdU-positive GSCs [E(Pc) KD testes: $20.3 \%(N=113)$ vs. Ctrl testes: $20.5 \%$ $(N=122)]$ and $\mathrm{pH} 3$-positive GSCs $[E(P c)$ KD testes: 2.02\% $(N=248)$ vs. Ctrl testes: $2.01 \%(N=199$ GSCs $)]$ were comparable, suggesting normal cell cycle progression of $E(P c)$ mutant GSCs.

\section{E(Pc) mutant germ cells accumulate DNA DSBs and have increased cell death}

We next examined whether $E(P c)$ mutant GSCs have increased cell death using anti-phosphorylated $\mathrm{H} 2 \mathrm{Av}$ $(\gamma \mathrm{H} 2 \mathrm{Av})$ for immunostaining. Increased $\gamma \mathrm{H} 2 \mathrm{Av}$ was detected in $E(P c)$ mutant $(N=18)$ compared with $C t r l$ GSCs (Figs. 6a-d, compare the GSCs indicated by yellow $v s$. green arrowheads in Figs. 6d, m), and in $E(P c)$ spermatogonial clones $(N=31$, Figs. 6e-h). Furthermore, increased $\gamma \mathrm{H} 2 \mathrm{Av}$ signal did not arise from increased $\mathrm{H} 2 \mathrm{Av}$ (Figs. 6i-1, compare the GSCs indicated by yellow $v s$. green arrowheads in Figs. 61, m). Therefore, $E(P c)$ mutant germ cells failed to exchange $\gamma \mathrm{H} 2 \mathrm{Av}$ with unphosphorylated $\mathrm{H} 2 \mathrm{Av}$, likely leading to accumulation of DNA DSBs. 
Fig. $4 \mathrm{E}(\mathrm{Pc})$ in germ cells is required for the expression of differentiation factor Bam. Apical tip of the wild-type testes a-d and $t u b-G a l 80^{t s}$, nos $>E$ $(P c)$ shRNA $[E(P c) \mathrm{KD}]$ testes e-h immunostained with DAPI, Vasa and Bam. Higher magnification of Bam-C staining at the apical tip shown in c', g' and k'. Asterisk: hub. Scale bar: $20 \mu \mathrm{m}$. i-l Apical tip of testes immunostained with GFP, DAPI, and Bam. GFP is used to identify $E(P c)$ mutant clones. Asterisk: hub. Scale bar: $50 \mu \mathrm{m}$. m Percentage of testes with excess germ cells. $* * * * P<$ 0.0001, Chi-square test. $\mathbf{n}$ Quantitative RT-PCR of bam mRNA in tub-Gal80 ${ }^{\text {ts }}$, nos-Gal4 $(C t r l)$ and $t u b-G a l 80^{t s}$, nos $>E$ $(P c)$ shRNA $[E(P c) \mathrm{KD}]$ testes. Mean \pm SD, based on three independent biological replicates. Transcript level in $C t r l$ testes set to 1 . Student's $t$ test. o A genome browser snapshot of the bam gene region. Compared with input, no enrichment of $\mathrm{E}(\mathrm{Pc})$ at genomic region of bam. The black lines indicate the average read density at chromosome 3R. Two independent replicates are shown here.
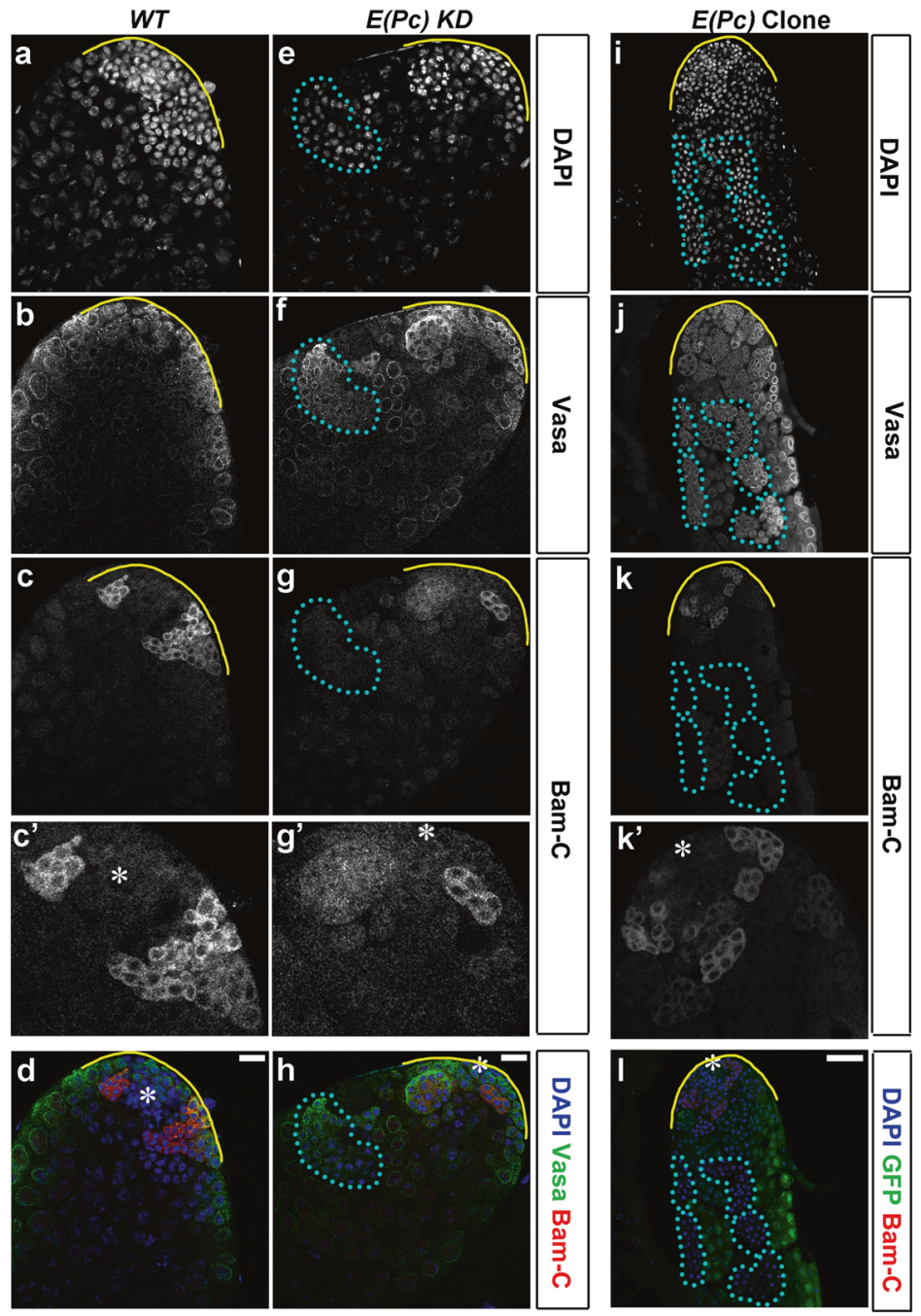

m

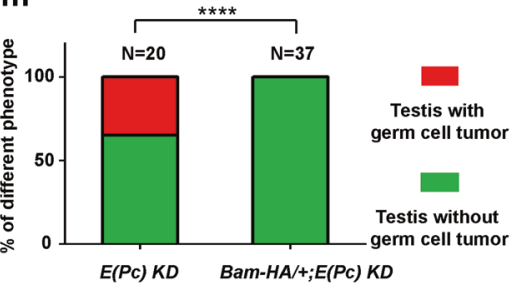

n

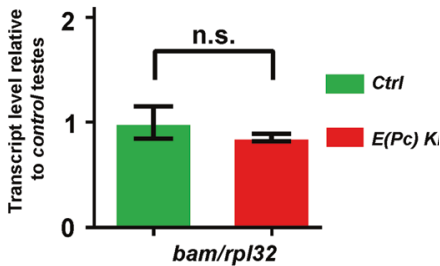

o

10

E(Pc) ChIP 1

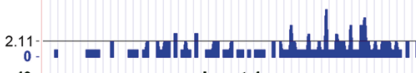

Input 1 1.19
10

1.70
$\mathbf{1 0}$

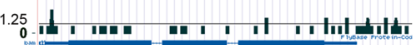

To further investigate this possibility, endonuclease I-CreI was expressed using heat shock (hs) promoter (hs-I-Crel) to introduce ectopic DNA DSBs at $18 \mathrm{~s}$ ribosomal gene repeats [38]. Meanwhile, $E(P c) \mathrm{KD}$ was temporally controlled using the tub-Gal80 ${ }^{t s}$, nos $>E(P c)$ shRNA strain (as in Figs. 1, 2, 4). 
Fig. $5 E(P c)$ mutant GSC clones have unchanged JAK-STAT and BMP signaling activity, as well as normal DE-Cad level. a-i Apical tip of testes immunostained with GFP a, d, $\mathbf{g}$ to identify $E(P c)$ mutant clones (yellow arrowhead) and Ctrl cells (green arrowhead). Vasa b, $\mathbf{e}, \mathbf{h}$ is used to identify germ cells. Immunostaining with STAT92E $\mathbf{c}, \mathrm{pMad} \mathbf{f}$ and DECad $\mathbf{i}$ in $E(P c)$ mutant GSC clones and Ctrl GSCs shows no difference. Hub: asterisk. Scale bar: $20 \mu \mathrm{m}$.
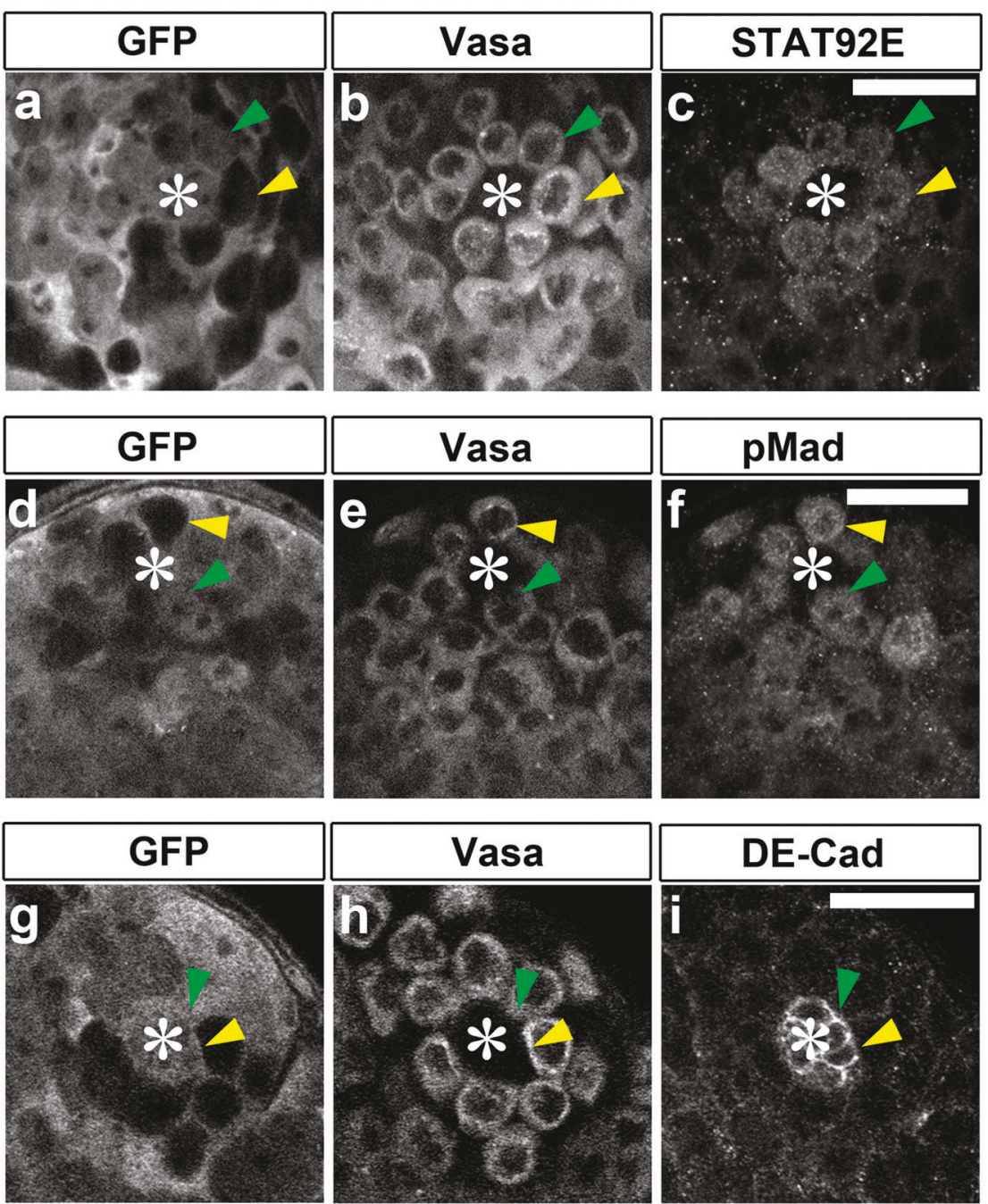

Without heat shock, $\gamma \mathrm{H} 2 \mathrm{Av}$ signal was detected in very few germline cysts (<5/testis) in both hs-I-CreI (Ctrl, Fig. 6n) and hs-I-CreI, tub-Gal80 ${ }^{t s}$, nos $>E(P c)$ shRNA $[E$ $(P c) \mathrm{KD}$ ] testes under permissive condition (Fig. 6q). By contrast, robust $\gamma \mathrm{H} 2 \mathrm{Av}$ signal was detected in all germ cells $12 \mathrm{~h}$ after inducing I-CreI expression in both Ctrl (Fig. 6o) and $E(P c) \mathrm{KD}$ (Fig. 6r) testes. After a 3-day recovery, DNA damage was efficiently repaired in most Ctrl (Fig. 6p) but not in $E(P c) \mathrm{KD}$ (Fig. 6s) testes. Under this condition, only $16 \%$ of $C t r l$ testes $(N=24)$ were found to be devoid of germ cells, whereas $52 \%$ of $E(P c)$ KD testes $(N=27)$ had no germ cells. These results suggest that the germline KD of $E(P c)$ caused failure in DNA damage repair and germ cell loss.

Moreover, neither tub-Gal80 ${ }^{t s}$, nos-Gal4 nor tub-Gal80 ${ }^{t s}$, nos $>E(P c)$ shRNA control testes had detectable $\gamma \mathrm{H} 2 \mathrm{Av}$ using a similar regime (Supplementary Fig. 6). For tubGal80 ${ }^{t s}$, nos $>E(P c)$ shRNA testes, the lack of detectable $\gamma \mathrm{H} 2 \mathrm{Av}$ likely resulted from partial retention of $\mathrm{E}(\mathrm{Pc})$ activity with a short time ( $\leq 3$ days) under the restrictive condition. These results suggest that the differences in $\gamma \mathrm{H} 2 \mathrm{Av}$ signals (Figs. 6o-p vs. r-s) likely resulted from the failure to repair I-CreI-induced DNA damage in $E(P c) \mathrm{KD}$ germ cells.

We next explored potential mechanisms underlying increased cell death in $E(P c)$ loss-of-function germ cells. In response to DNA DSBs, Chk2, a checkpoint kinase, is activated by ataxia-telangiectasia mutated kinase to regulate cell cycle arrest, DNA repair and apoptosis [39]. Recent studies revealed that DNA damage induces Drosophila female GSC loss, which could be rescued by reducing Chk2 [40]. Therefore, we tested the potential roles of Chk2 in regulating germ cell loss in $E(P c) \mathrm{KD}$ testes. To enhance the KD effect, both $C t r l$ and $E(P c)$ KD males were grown at 29 ${ }^{\circ} \mathrm{C}$, under which condition $100 \%$ of $\mathrm{Ctrl}$ testes showed normal morphology (Fig. 7a), whereas $85 \%$ of $E(P c) \mathrm{KD}$ testes $(N=48)$ were completely devoid of germ cells (Fig. 7b) and 15\% had fewer than 40 germ cells (Fig. 7c). Significant rescue of the germ cell loss $\left(P<10^{-4}\right)$ was observed when chk2, but not the control mCherry, was 
Fig. $6 E(P c)$ germline clones exhibit increased $\gamma \mathrm{H} 2 \mathrm{Av}$. a-l Apical tip of testes stained with GFP $\mathbf{b}, \mathbf{f}, \mathbf{j}$ to identify GFPnegative $E(P c)$ mutant clones, DAPI $\mathbf{c}, \mathbf{g}, \mathbf{k}, \gamma \mathrm{H} 2 \mathrm{Av} \mathbf{d}, \mathbf{h}$ and H2Av l. m Quantification of intensity of $\gamma \mathrm{H} 2 \mathrm{Av}$ and $\mathrm{H} 2 \mathrm{Av}$ signals between GFP-negative $E$ (Pc) mutant clones and GFPpositive control germ cells. $\gamma \mathrm{H} 2 \mathrm{Av}^{\text {clone }} / \gamma \mathrm{H} 2 \mathrm{Av}^{\text {ctrl }}=4.05 \pm$ 2.58. $\mathrm{H} 2 \mathrm{Av}^{\text {clone }} / \mathrm{H} 2 \mathrm{Av}^{\text {ctrl }}=1.00$ \pm 0.33 (mean $\pm \mathrm{SD})$. Two-tailed $t$-test. $* * * * P<0.0001$. $\mathbf{n}-\mathbf{p}$ Apical tip of $h s-I-C r e I$ testes before heat shock (no HS) n, a half day after heat shock $(0.5 \mathrm{D}$ AHS) $\mathbf{o}$ and 3 days after heat shock (3D AHS) p stained with hub and cyst cell marker Arm and DNA DSB marker $\gamma \mathrm{H} 2 \mathrm{Av}$. q-s Apical tip of hs-I-CreI, tub$\mathrm{Gal}_{80} 0^{t s}$, nos $>E(P c)$ shRNA testes before heat shock $\mathbf{q}, 0.5 \mathrm{D}$ AHS $\mathbf{r}$, and 3D AHS s stained with Arm and $\gamma \mathrm{H} 2 \mathrm{Av}$. Asterisk: hub. Scale bar: $20 \mu \mathrm{m}$
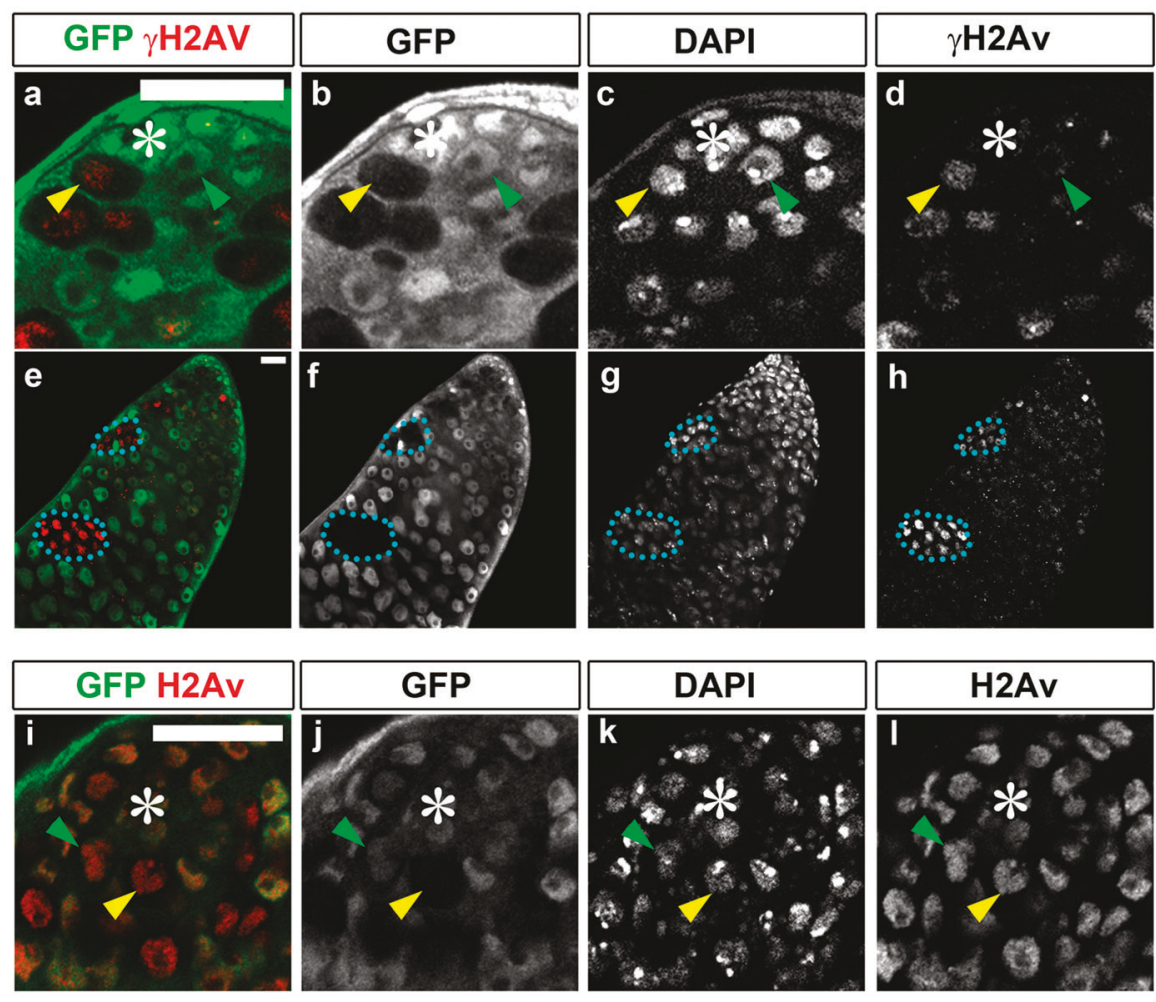

m

Intensity ratio: $E(P c)$ clone vs. Heterozygous cells
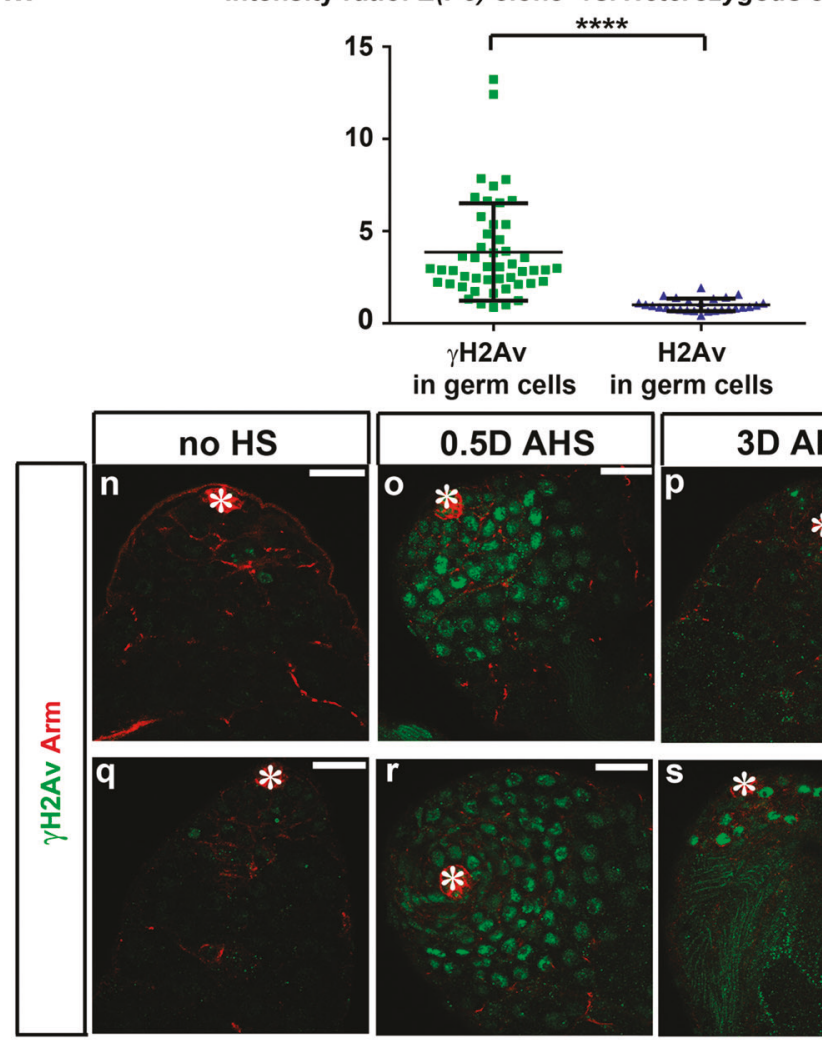

in germ cells in germ cells

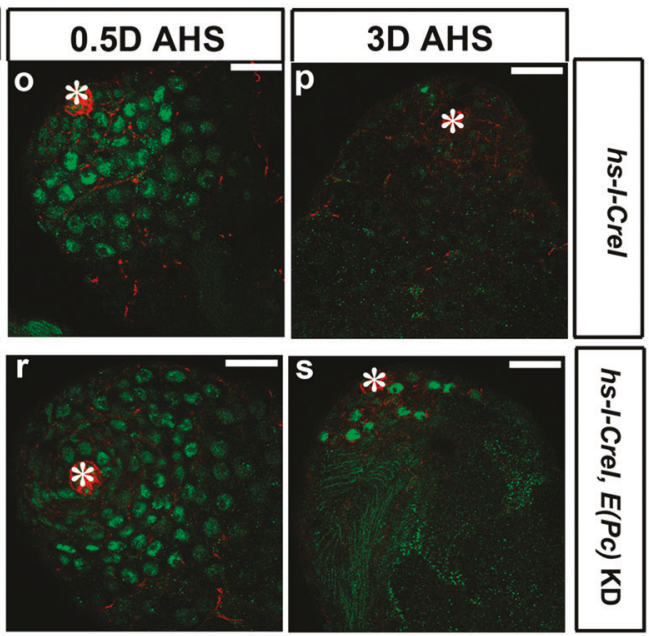

compromised in $E(P c) \mathrm{KD}$ testes (Figs. 7d-k). Quantification showed increased GSC number in $c h k 2, E(P c)$ double $\mathrm{KD}$ testes compared with $E(P c)$ single $\mathrm{KD}$ testes, but since chk2 KD itself led to GSC increase, this readout could be additive (Fig. 71). Moreover, in these experiments because both $\mathrm{E}(\mathrm{Pc})$ and Chk2 were inactivated throughout Drosophila development, the overall readout could arise from either establishment or maintenance effects, or both. 


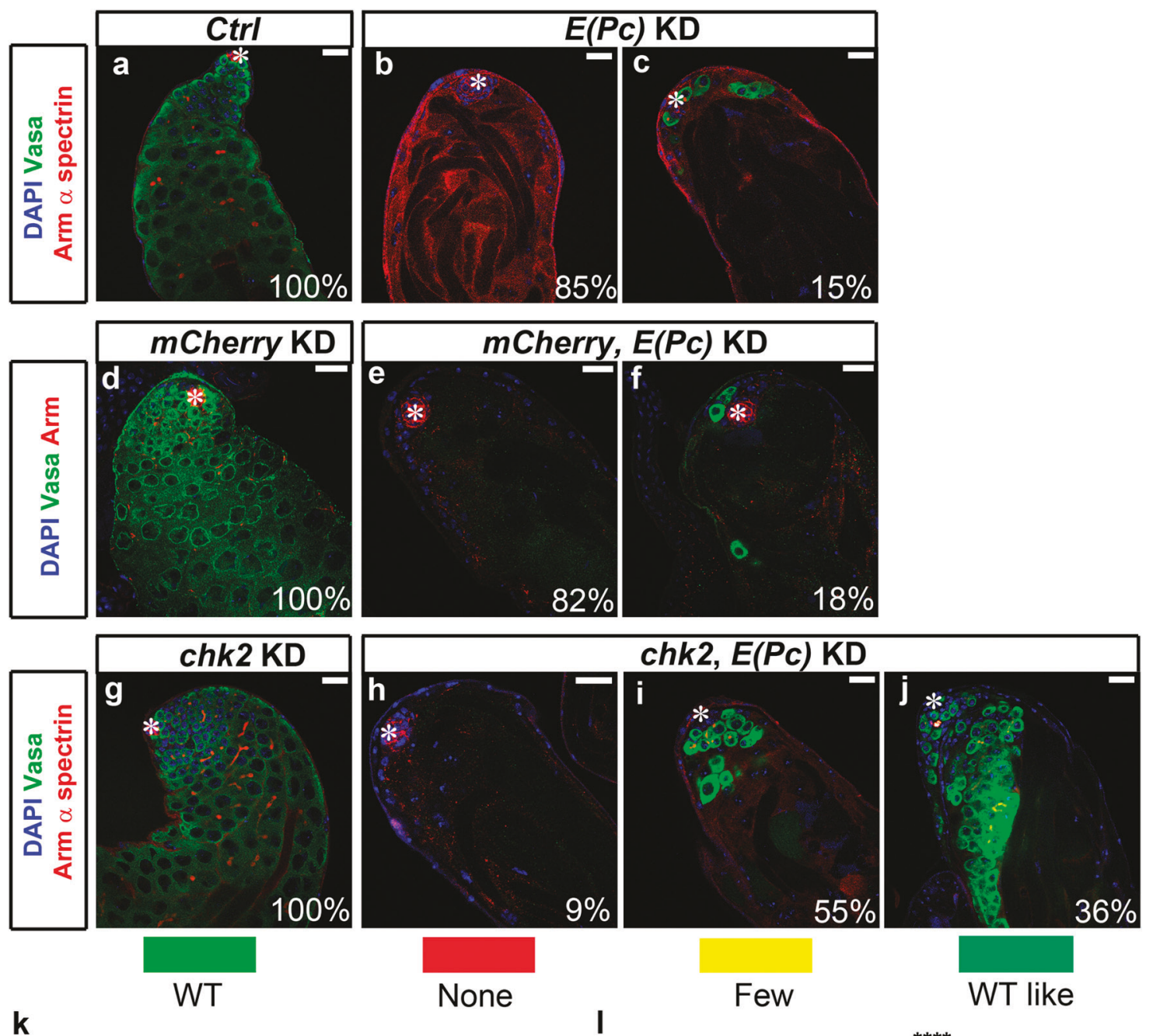

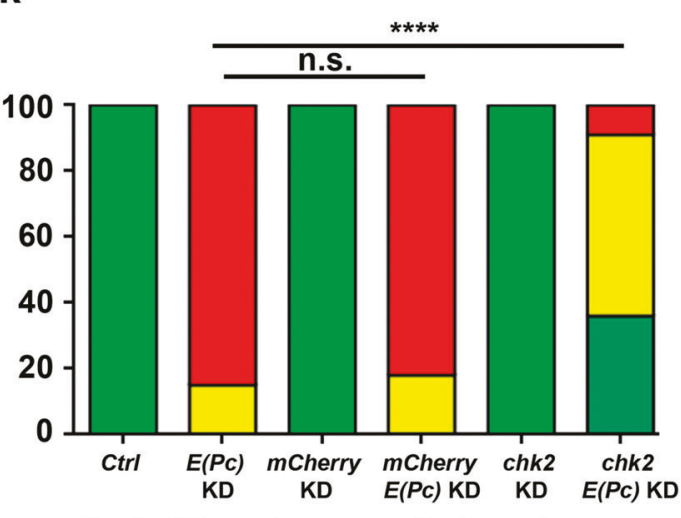

$\%$ of different germ cell phenotype

Fig. 7 CHK2 mediates germ cell loss induced by $E(P c)$ knockdown. a-j Control (Ctrl) [nos-Gal4/+], E(Pc) KD [nos $>E(P c)$ shRNA], $m$ Cherry KD [nos $>m$ Cherry shRNA], mCherry, E(Pc) KD [nos $>$ $m$ Cherry shRNA, E(Pc) shRNA], chk2 KD [nos >chk2 shRNA], and chk2, $E(P c) \mathrm{KD}[$ nos > chk2 shRNA, E(Pc) shRNA] testes stained with

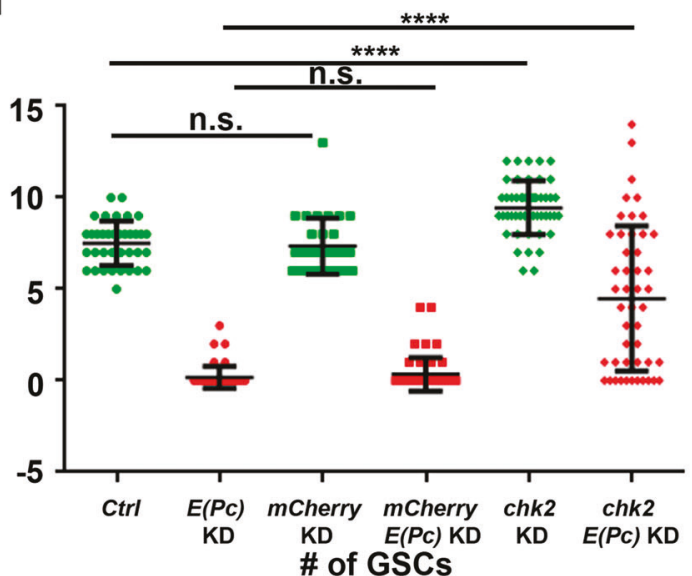

DAPI, germ cell marker Vasa, hub and cyst cell marker Arm, and fusome marker $\alpha$-spectrin. Asterisk: hub. Scale bar: $20 \mu \mathrm{m}$. k Quantification of germ cell phenotype. $* * * * P<0.0001$. Chi-square test. 1 Quantification of GSCs number (average \pm SD). Mann-Whitney $U$ test. $* * * P<0.0001$. 

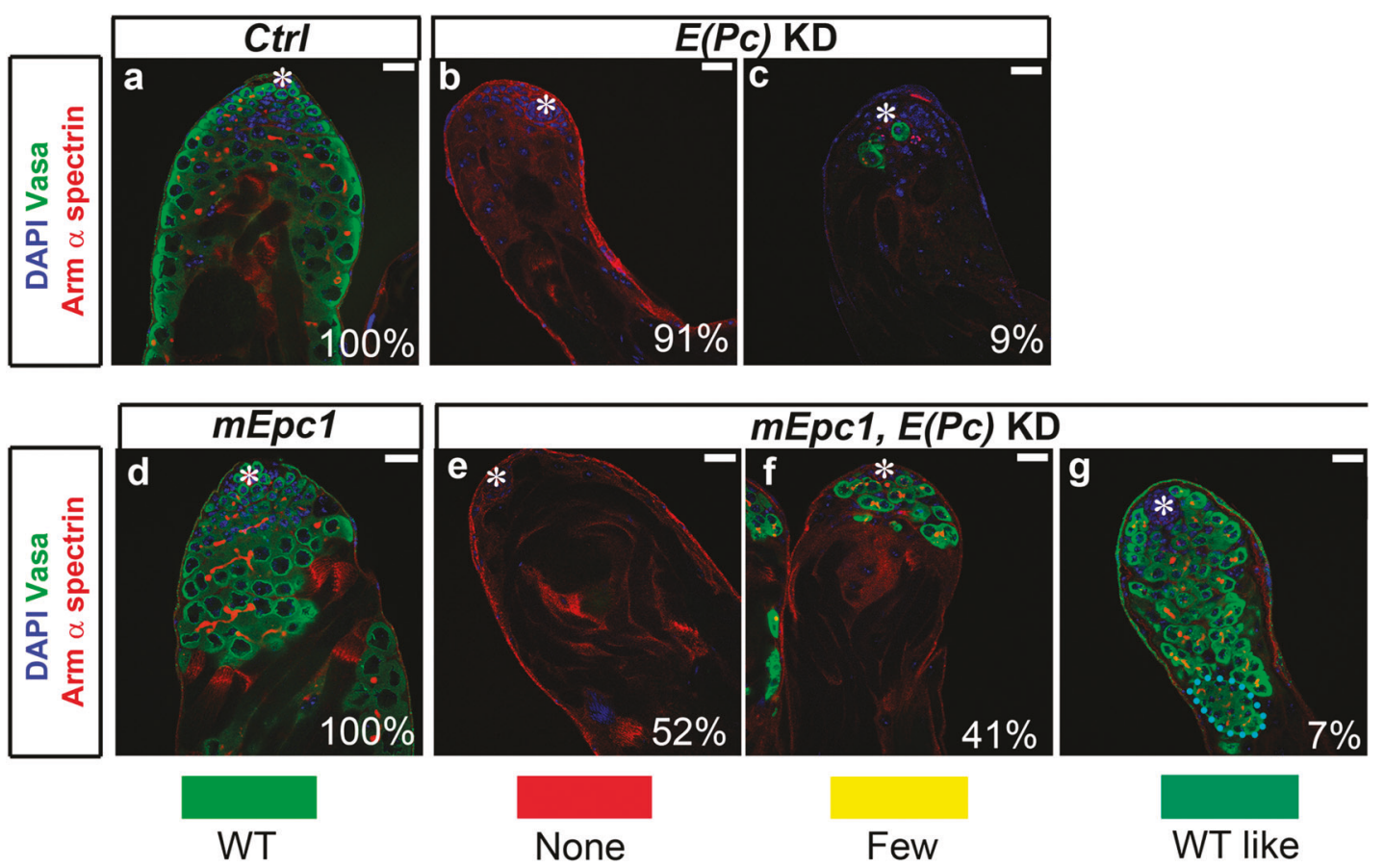

h

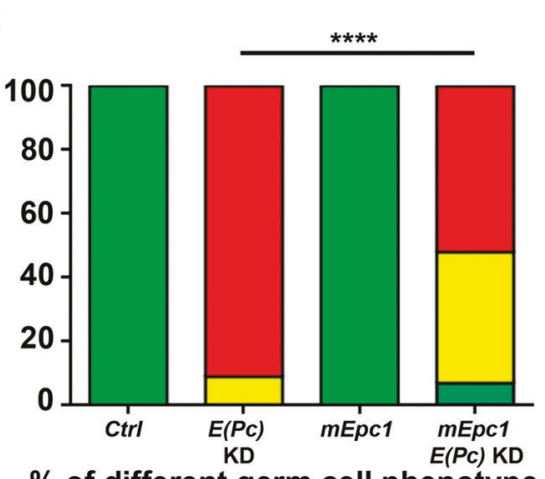

i

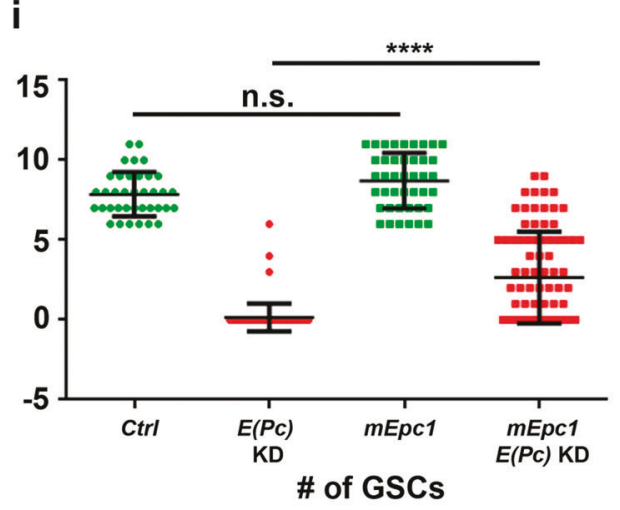

$\%$ of different germ cell phenotype

SB

j

(2) repair DNA damage and prevent $\mathrm{CHK} 2$ dependent Germ cell death germ cell loss

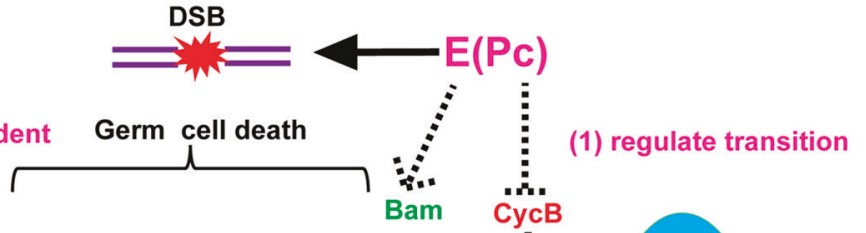

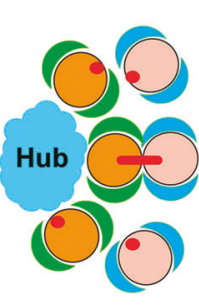

GSCs

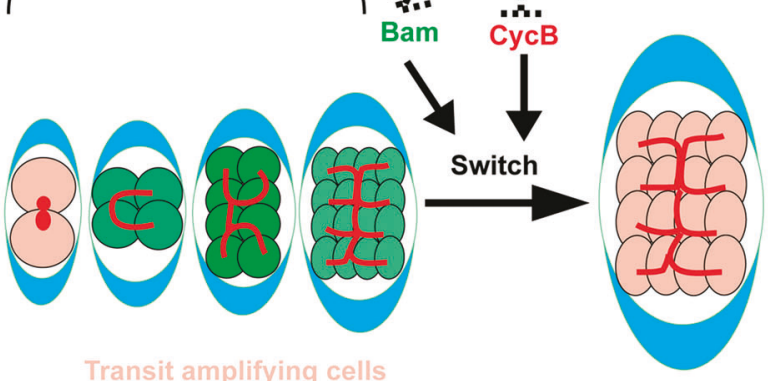

Primary

Spermatocyte 
Fig. 8 Expression of $\mathrm{mEpc} 1$, mouse homolog of $\mathrm{E}(\mathrm{Pc})$, partially complements the germ cell loss phenotype in $E(P c) \mathrm{KD}$ testes. $\mathbf{a}-\mathbf{g}$ Testes from control (Ctrl) $[$ nos-Gal4/+], E(Pc) KD [nos $>E(P c)$ shRNA], germline expression of $m E p c 1$ [nos $>m E p c l]$ and germline expression of $m E p c l$ at $E(P c) \mathrm{KD}$ background [nos $>m E p c 1, E(P c)$ shRNA] males stained with DAPI, germ cell marker Vasa, hub and cyst marker Arm, and fusome marker $\alpha$-spectrin. Hub: asterisk. Scale bar: $20 \mu \mathrm{m}$. h Quantification of germ cell loss phenotype. $* * * * P<0.0001$. Chi-square test. i Quantification of GSCs number (average \pm SD). Mann-Whitney $U$-test. $* * * * P<0.0001$. j A model depicting $\mathrm{E}(\mathrm{Pc})$ regulation of Drosophila male germ cell differentiation and maintenance. During spermatogenesis, the switch from mitosis-to-meiosis requires reduced cell cycle regulator $\mathrm{CycB}$ and accumulation of the differentiation factor $\mathrm{Bam}$. $\mathrm{E}(\mathrm{Pc})$ represses $C y c B$ transcription and positively regulates Bam protein accumulation to promote the mitosisto-meiosis transition (1). In addition, efficient DNA DSB repair to avoid spermatogonial cell death also requires $\mathrm{E}(\mathrm{Pc})(2)$.

\section{Functional conservation between Drosophila $\mathrm{E}(\mathrm{Pc})$ and mammalian homolog}

The $E(P c)$ gene is conserved from yeast to mammals, with two mouse homologs, Epc1 and Epc2. Epc1, compared with Epc2, shares more identity and similarity to the Drosophila $\mathrm{E}(\mathrm{Pc})[20]$. When mouse Epc1 was expressed in germ cells with compromised $E(P c)$, significant $\left(P<10^{-4}\right)$ rescue of the germ cell loss phenotype was observed (Figs. 8a-i), suggesting functional conservation between Drosophila E (Pc) and mouse Epc1.

\section{Discussion}

We herein report the critical roles of a chromatin factor E (Pc) in regulating GSC differentiation by targeting two critical genes. First, $\mathrm{E}(\mathrm{Pc})$ binds to the genomic region of $C y c B$ gene in $\mathrm{S} 2$ cells and negatively regulates $C y c B$ transcription in the germline, possibly through promoting $\mathrm{H} 4$ acetylation at $C y c B$ locus. Second, $\mathrm{E}(\mathrm{Pc})$ is required for proper accumulation of Bam protein in spermatogonia to promote the mitosis-to-meiosis transition. Moreover, $\mathrm{E}(\mathrm{Pc})$ is needed for effective DNA DSB repair in germ cells (Fig. 8j). Together, $\mathrm{E}(\mathrm{Pc})$ has pleiotropic roles in the male germline and such functions of $\mathrm{E}(\mathrm{Pc})$ could be cell type and stage specific.

Previous studies have shown transcriptional and posttranscriptional regulation of $C y c B$ in meiotic germ cells [23, $24,41,42]$. Here, our studies have identified $\mathrm{E}(\mathrm{Pc})$ as an upstream regulator of $C y c B$ through modulating $\mathrm{H} 4$ acetylation. Histone acetylation is normally linked to gene activation. However, in $E(P c)$ mutant germ cells, decreased $\mathrm{H} 4$ acetylation was accompanied by increased $C y c B$ expression, indicating a potential repressive role of $\mathrm{H} 4$ acetylation, as reported previously. For example, when $E$ $(P c)$ or Tip60 is knocked down in cyst cells of Drosophila testes, both zfh-1 and yan are upregulated [21]. Moreover, $\mathrm{H} 3 \mathrm{~K} 56 \mathrm{ac}$ was found to repress transcription of newly replicated DNA in budding yeast [43]. Noticeably, a recent study revealed that expression of the dominant negative $d T i p 60^{E 431 Q}$ or $\mathrm{KD}$ of $E(P c)$ reduced $C y c B$ expression in Drosophila wing disc, suggesting that the regulation of dTip60 and $\mathrm{E}(\mathrm{Pc})$ on $C y c B$ could be cell type and/or developmental stage dependent [44].

Bam is both necessary and sufficient for male germline differentiation and exhibits a highly orchestrated spatiotemporal expression pattern, which is regulated by transcriptional, post-transcriptional and protein stability mechanisms [2, 6, 7, 42, 45]. Here, we found that $\mathrm{E}(\mathrm{Pc})$ regulates Bam protein accumulation via an unknown posttranscriptional mechanism(s). A recent study in Drosophila female GSC lineage demonstrates that Bam is a component of a deubiquitinase complex and that Bam-dependent deubiquitination stabilizes cyclin A (CycA) [46], suggesting a connection between Bam and cell cycle genes.

Germ cells have a unique quality control mode for survival [10-12]. Our studies suggest that $\mathrm{E}(\mathrm{Pc})$ could participate in such a control. In Drosophila testis, GSCs, gonialblasts, and spermatogonia undergo DNA replication and mitosis. Replication stress, such as replication fork collapse caused by unrepaired DNA lesion, could cause DNA DSBs. Indeed, testes from flies fed with the replicative stress-inducing agent hydroxyurea have intensive $\gamma \mathrm{H} 2 \mathrm{Av}$ signals in mitotic germ cells [47]. Under normal conditions, lack of $\gamma \mathrm{H} 2 \mathrm{Av}$ signal in the germline results from robust DNA repair. Under both physiological conditions and induction of ectopic DNA damage with endonuclease expression, compromising $\mathrm{E}(\mathrm{Pc})$ in germ cells led to accumulated DNA damage, demonstrated by robust $\gamma \mathrm{H} 2 \mathrm{Av}$ and severe germ cell loss. Reminiscent of the roles of $E(P c)$ in the male germline, female germline with compromised $E(P c)$ produce no mature eggs and have severe cell death [48], suggesting similar requirement of $\mathrm{E}(\mathrm{Pc})$ for both male and female germ cell survival.

Abnormal activity of the human $\mathrm{E}(\mathrm{Pc})$ homolog EPC1 leads to T-cell leukemia/lymphoma [49]. Moreover, analysis of 99 pancreatic tumors has identified significantly higher mutation rate at the Epcl locus, suggesting a potential tumor-suppressor role of EPC1 in pancreas [50]. However, the in vivo roles of $\mathrm{E}(\mathrm{Pc})$ remain unclear. Here, our studies provide insights into the molecular and cellular mechanisms of $\mathrm{E}(\mathrm{Pc})$ in a well-established adult stem cell system, which will help understanding its normal functions and relationship with diseases.

\section{Materials and methods}

\section{Fly strains and husbandry}

Fly stocks were raised under standard yeast/molasses medium at $25^{\circ} \mathrm{C}$ unless stated otherwise. The following 
flies were used: $E(P c)^{l}$ (Bloomington Drosophila Stock Center, BL3056), $E(P c)^{w 3}$ (BL9396), UAS-E(Pc) gDNA$G F P$ and $U A S-E(P c)$ cDNA-GFP [21], UAS-E(Pc) shRNA (BL35271), UAS-dTip60 ${ }^{E 431 Q}$ (from Felice Elefant, Drexel University, Philadelphia, Pennsylvania, USA), UAS-chk2 shRNA (BL35152), UAS-mCherry shRNA (from Mark Van Doren, Johns Hopkins University, Baltimore, Maryland, USA), hs-I-CreI [51], nos-Gal4/Cyo [32], nos-Gal4/Cyo; tub-Gal80 ${ }^{t s} / T M 6 B$ (from Yukiko Yamashita, University of Michigan, Ann Arbor, Michigan, USA), bam-HA [6], FRT42B (BL1956), $C y c B^{2} / C y o$ (BL6630).

To study $\mathrm{E}(\mathrm{Pc})$ and Tip60 function in germ cells, UAS-E (Pc) ShRNA, and UAS-dTip60 ${ }^{E 431 Q}$ were crossed with nosGal4/Cyo at $25^{\circ} \mathrm{C}$ separately. To study intermediate germ cell phenotype induced by $E(P c) \mathrm{KD}$, flies with the following genotype were generated: nos-Gal4/Cyo;tub$G a l 80^{t s} / U A S-E(P c) s h R N A$. One-day-old males were shifted to $29^{\circ} \mathrm{C}$ to induce expression of $E(P c) s h R N A$ by inacti-

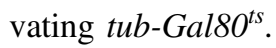

To explore if loss of $\mathrm{CycB}$ or increased Bam could rescue the overproliferative spermatogonial tumor phenotype induced by $E(P c) \mathrm{KD}$ in germ cells, the following flies were generated: nos-Gal4/CycB ${ }^{2} ; U A S-E(P c) s h R N A / t u b-$

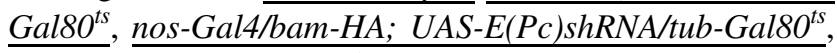
nos-Gal4/Cyo;tub-Gal80 $0^{t s} / U A S-E(P c) s h R N A$. One-day-old males from $18^{\circ} \mathrm{C}$ were collected and shifted to $29^{\circ} \mathrm{C}$.

To investigate if expression of mEPc1 could rescue the $E$ $(P c) \mathrm{KD}$ germ cell loss phenotype, the following fly strains were generated: nos-Gal4/+, nos-Gal4/+; UAS-E(Pc) shRNA/+, nos-Gal4/UASp-mEpcl complementary DNA (cDNA), nos-Gal4/UASp-mEpcl cDNA; UAS-E(Pc)shRNA/ \pm . One-day-old males were shifted to $29^{\circ} \mathrm{C}$ to enhance $E$ $(P c)$ shRNA KD effects, followed by dissection at 5 days.

To investigate the roles of Chk2 in $E(P c) \mathrm{KD}$ testes, the following flies were generated: nos-Gal4/+, nos-Gal4/+; $\underline{U A S-E(P c) \operatorname{shRNA/+}, \text { nos-Gal4/+; UAS-Chk2 shRNA/+ }}$ nos-Gal4/+; UAS-Chk2 shRNA/UAS-E(Pc) shRNA, nosGal4/+; UAS-mCherry shRNA/+, nos-Gal4/+; UASmCherry shRNA/ UAS-E(PC) shRNA . One-day-old males were shifted to $29^{\circ} \mathrm{C}$ to enhance $E(P c) s h R N A$ KD effects, followed by dissection at 5 days.

To induce DNA damage, 1-day-old $\mathrm{hs}$-I-CreI males were heat shocked at $37^{\circ} \mathrm{C}$ for $30 \mathrm{~min}$ and then transferred to 29 ${ }^{\circ} \mathrm{C}$ for half a day or 3 days before dissection. To investigate the roles of $\mathrm{E}(\mathrm{Pc})$ in DNA damage repair, the following flies

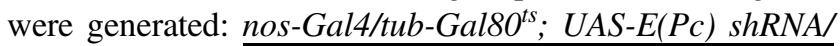
hs-I-CreI, nos-Gal4/tub-Gal80 ${ }^{\text {ts }}$; hs-I-CreI/+, nos-Gal4/

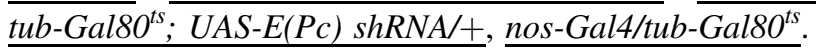

\section{Clonal analysis}

To generate $E(P c)$ clones, either $E(P c)^{w 3}$ or $E(P c)^{1}$ allele was recombined with $F R T 42 B$ to generate $F R T 42 B, E(P c)$
${ }^{w 3} / C y o$ or FRT42B, E(Pc) $)^{l} / C y o$ flies. Adult flies with the following genotypes were raised at $25{ }^{\circ} \mathrm{C}$ until pupal stage: $\underline{h s-F L P ; F R T 42 B}, \underline{U b i-G F P / F R T 42 B}, \underline{E(P c)^{w 3}}$ or hs-FLP; FRT42B, Ubi-GFP/FRT42B, $\underline{E(P c)^{l}}$ and control flies $\underline{h s-}$ FLP; FRT42B, Ubi-GFP/FRT42B. Then they were heat shocked for $2 \mathrm{~h}$ each on 2 consecutive days. One-day after heat shock, adult flies were collected and aged until dissection.

\section{Generation of transgenic fly lines}

For transgenic fly UASp-mEpcl $c D N A, E p c l$ was amplified using the mouse sperm cDNA library. The Epcl cDNA was amplified as a $K p n I$ and $X b a$ I flanked fragment with Epc1 F1 and R1 primers. Epcl was then ligated into pGEM-Teasy vector (Promega) followed by sequencing. The fulllength Epcl cDNA was retrieved with $K p n \mathrm{I}$ and $X b a \mathrm{I}$ and ligated into UASp vector cut with the same two enzymes. Transgenic fly lines were generated by Bestgene Inc. (Chino Hills, CA).

Primers:

Epc1 F1: 5'-CGGGGTACCATGGAACACCATCTTCAGCG-3';

Epc1 R1: 5'-TGCTCTAGATTACGTCACCTCCATTGCTACTGTG-3'.

\section{Immunofluorescence}

Testes were dissected and immunostained as previously described [52]. Primary antibodies were as follows: Vasa (rabbit, 1:200, Santa Cruz, sc-30,210), Vasa (rat, 1:50, DSHB), Fas III (mouse, 1:100, DSHB, 7G10), Armadillo (mouse, 1:200, DSHB, N2 7A1), $\alpha$-spectrin (mouse, 1:50, DSHB, 3A9), GFP (chicken, 1:1000, Abcam, ab13970), STAT92E (rabbit, 1:800, from Denise Montell, UC Santa Barbara), pMAD (rabbit, 1:100, Abcam, ab52903), DE-Cad (rat, 1:20, DSHB, DCAD2), anti-Ser10-phosphorylated Histone H3 (mouse, 1:1000, Millipore, \#05-806), H3K27me3 (rabbit, 1:2000, Millipore, \#07-449), H3K4me3 (rabbit, 1:2000, Millipore, \#07-473), H2Av (rabbit, 1:500, active motif, 39,716), $\gamma$-H2Av [53, 54] (pH2AvDS137, rabbit, 1:1000, Rockland, 600-401-914), H4 tetra-acetylation (rabbit, 1:500, from Keji Zhao, NHLBI, NIH), H4 (rabbit, 1:200, Abcam, ab10158), Bam-C (mouse, 1:20, DSHB, bam), CycB (mouse, 1:20, DSHB, F2F4). For $\mathrm{CycB}$ and Bam-C staining, testes were dissected in $1 \times$ phosphate-buffered saline and transferred onto slides. Slides were frozen in liquid nitrogen, followed by fixation in chilled methanol for $5 \mathrm{~min}$ and acetone for $2 \mathrm{~min}$. Then testes were incubated with primary antibodies as mentioned.

Secondary antibodies were all Alexa Fluor series (1:200, Molecular Probes). Images were taken with Zeiss 
LSM 510 META or LSM 700 using LSM software. Images were processed using Adobe Photoshop. Lysotracker (Invitrogen, L-7528) was used to label cells undergoing cell death, following the manufacturer's protocol. EdU incorporation was performed with the Click-iT EdU Alexa Fluor 488 Imaging Kit (Invitrogen C10083). Dissected testes were incubated with EdU solution for 30 min, followed by fixation and immunostaining steps, as described.

\section{ChIP-seq and data analysis}

To perform S2 cell ChIP, transfection with $1 \mu \mathrm{g}$ Actin-Gal4 and $1 \mu \mathrm{g} U A S-E(P c) c D N A-G F P$ plasmids was done following the manufacturer's protocol with Effectene Transfection Reagent (\#301425, Qiagen). S2 cells were split the day before the transfection experiment. On the day of transfection, $4 \times 10^{6}$ cells per $25 \mathrm{~cm}^{2}$ flask were seeded in $4 \mathrm{ml}$ growth medium containing serum and antibiotics. After transfection, S2 cells were incubated at room temperature for $40-48 \mathrm{~h}$ to obtain maximal level of gene expression. S2 cell fixation and subsequent ChIP were performed with Active Motif's ChIP-IT High Sensitivity Kit, following the manufacturer's instruction. Sonication of S2 cells was done with the Bioruptor UCD200 sonicator (Diagenode), using the following settings: 0.5 -min ON and 1-min OFF for a total of $15 \mathrm{~min}$. Fragment size was around 500-600 bp. ChIP-grade GFP antibody (Abcam, ab290) [21, 55-59] was used to pull down sheared chromatin.

Libraries were generated using reagents provided in the Illumina TruSeq ChIP Sample Preparation Kit (IP-2021012). The Illumina compatible libraries were sequenced with Hi-seq sequencer (Hi-Seq, Illumina). Then 75-bp pairend read sequencing was performed. FASTQ raw data files were filtered with the Fastqc quality control software (www. bioinformatics.babraham.ac.uk/projects/fastqc/). The BOWTIE program (version 0.12.7) was utilized to align reads to Drosophila genome (dm3), with the running parameters (bowtie -p 8 -t -a-phred33 -quals -n 2 -e 70 -1 48 -m 1-best-strata). Pair-end reads were treated as separate single reads. At each chromosome position, only one read was retained to get non-redundant read count data. SAM (Sequence Alignment/Map)-formatted alignment files will be uploaded onto NIH GEO database upon proper acceptance. Enrichment of reads across the genome was analyzed by MACS2 for peak calling, which was performed with paired experiment and control genome input under default parameter settings. UCSC genome browser customized visualization tools were also applied in the analysis. SAM tools software suite was utilized to convert between related read formats.

\section{ChIP quantitative PCR (qPCR)}

As previously described, a similar protocol was used for ChIP with $\mathrm{H} 4$ tetra-acetylation antibody (A gift from Keji Zhao, NHLBI, NIH) on 100 pairs of wild-type testes. The sonication setting was 0.5 -min ON and 1-min OFF repetitively for a total of $30 \mathrm{~min}$. qPCR was performed as previously described. Two independent biological replicates were used. Each PCR reaction was performed in duplicate, and average $\mathrm{Ct}$ values were used.

Primers used for $C y c B$ qPCR were as follows:

P5 forward: 5'-AGTCCCTTGAGCAGTTTTCG-3',

P5 reverse: 5'-CTCCAGTTTAGTTCGGTTCCTAG-3';

P4 forward: 5'-AGAAAGAGTGCCGTTTGTCC-3',

P4 reverse: 5'-ATTGCGCCAAAATGTGAGC-3';

P3 forward: 5'-TCGGAGAATTGACAATCCCG-3',

P3 reverse: 5'-AGACGCTTGGCTATCACTTG-3';

P2 forward: 5'-ATATGCTAATGAGCCAGAGCG-3',

P2 reverse: 5'-GCCATCGCCTCAGAATTTTG-3';

P1 forward: 5'-CTTCCTTATGGATCGACACTCG-3',

P1 reverse: 5'-TGCGTTTAAATGACAAAAGACTGG-3'.

\section{Quantitative RT-PCR}

The cDNA preparation of testes and RT-PCR were performed and analyzed as previously described. Each PCR reaction was performed in triplicate, and $\mathrm{Ct}$ values were averaged. Three biological replicates were used.

Primers:

bam forward: 5'-ACTCAGCGCATGGAGAGATTGCTA-3',

bam reverse: 5'-AGTAGCGGTGCTCCAGATCCATTT-3';

CycB forward: 5'-CTGTTCGTTTCGTGTTCGTTAAA-3',

CycB reverse: 5'-CAAGGGACTCCAGCAGATTAC-3'; rpl32 forward: 5'-CATGCTGCCCACCGGATTCAAGAAG-3',

rpl32 reverse: 5'-CTCGTTCTCTTGAGAACGCAGGCGA-3'.

\section{Quantification of immunofluorescent signal intensity}

Fluorescence intensity between the GFP-positive control cells and GFP-negative $E(P c)$ mutant cells in the same testes was quantified using ImageJ software. Data were analyzed and presented using GraphPad Prism software.

\section{Data availability}

GEO accession number for ChIP-seq data is GSE98484. 
Acknowledgements We thank Drs. M. Van Doren, D. DrummondBarbosa, R. Johnston, and Chen lab members for critical comments. We thank Dr. F. Elefant for the UAS-dTip60 ${ }^{E 431 Q}$ Drosophila line, Dr. Y. Yamashita for the nos-Gal4/Cyo;tub-Gal80 ${ }^{\text {ts }} / T M 6 B$ Drosophila line, Dr. Ting Xie for the hs-I-CreI Drosophila line, TRiP at Harvard Medical School (NIH/NIGMS R01-GM084947) for providing transgenic RNAi fly stocks used in this study, Keji Zhao for anti-ace H4 antibodies, and the Developmental Studies Hybridoma Bank for antibodies. Stocks obtained from the Bloomington Drosophila Stock Center (NIH P40OD018537) were used in this study. This study was supported by NIH RO1HD065816, the David and Lucile Packard Foundation, Howard Hughes Medical Institute, Bill \& Melinda Gates Foundation and the Simons Foundation, as well as Johns Hopkins University start-up (XC).

\section{Compliance with ethical standards}

Conflict of interest The authors declare that they have no conflict of interest.

\section{References}

1. Morrison SJ, Spradling AC. Stem cells and niches: mechanisms that promote stem cell maintenance throughout life. Cell. 2008;132:598-611.

2. Insco ML, Leon A, Tam CH, McKearin DM, Fuller MT. Accumulation of a differentiation regulator specifies transit amplifying division number in an adult stem cell lineage. Proc Natl Acad Sci USA. 2009;106:22311-6.

3. Gonczy P, Matunis E, DiNardo S. Bag-of-marbles and benign gonial cell neoplasm act in the germline to restrict proliferation during Drosophila spermatogenesis. Development. 1997; 124:4361-71.

4. McKearin DM, Spradling AC. Bag-of-marbles: a Drosophila gene required to initiate both male and female gametogenesis. Genes Dev. 1990;4:2242-51.

5. Schulz C, et al. A misexpression screen reveals effects of bag-ofmarbles and TGF beta class signaling on the Drosophila male germ-line stem cell lineage. Genetics. 2004;167:707-23.

6. Eun SH, et al. MicroRNAs downregulate Bag of marbles to ensure proper terminal differentiation in the Drosophila male germline. Development. 2013;140:23-30.

7. Insco ML, et al. A self-limiting switch based on translational control regulates the transition from proliferation to differentiation in an adult stem cell lineage. Cell Stem Cell. 2012;11:689-700.

8. Feng L, Chen X. Epigenetic regulation of germ cells-remember or forget? Curr Opin Genet Dev. 2015;31:20-27.

9. Gan Q, et al. Dynamic regulation of alternative splicing and chromatin structure in Drosophila gonads revealed by RNA-seq. Cell Res. 2010;20:763-83.

10. Yacobi-Sharon K, Namdar Y, Arama E. Alternative germ cell death pathway in Drosophila involves HtrA2/Omi, lysosomes, and a caspase-9 counterpart. Dev Cell. 2013;25:29-42.

11. Yang H, Yamashita YM. The regulated elimination of transitamplifying cells preserves tissue homeostasis during protein starvation in Drosophila testis. Development. 2015;142:1756-66

12. Hasan S, Hetie P, Matunis EL. Niche signaling promotes stem cell survival in the Drosophila testis via the JAK-STAT target DIAP1. Dev Biol. 2015;404:27-39.

13. Bailly A, Gartner A. Germ cell apoptosis and DNA damage responses. Adv Exp Med Biol. 2013;757:249-76.

14. Bonner WM, et al. GammaH2AX and cancer. Nat Rev Cancer. 2008;8:957-67.
15. Ikura T, et al. Involvement of the TIP60 histone acetylase complex in DNA repair and apoptosis. Cell. 2000;102:463-73.

16. Squatrito M, Gorrini C, Amati B. Tip60 in DNA damage response and growth control: many tricks in one HAT. Trends Cell Biol. 2006; $16: 433-42$.

17. Kim $\mathrm{CH}$, et al. The chromodomain-containing histone acetyltransferase TIP60 acts as a code reader, recognizing the epigenetic codes for initiating transcription. Biosci Biotechnol Biochem. 2015;79:532-8.

18. Kusch T, et al. Acetylation by Tip60 is required for selective histone variant exchange at DNA lesions. Science. 2004;306:2084-7.

19. Sinclair DA, et al. Enhancer of polycomb is a suppressor of position-effect variegation in Drosophila melanogaster. Genetics. 1998;148:211-20.

20. Stankunas K, et al. The enhancer of polycomb gene of Drosophila encodes a chromatin protein conserved in yeast and mammals. Development. 1998;125:4055-66.

21. Feng L, Shi Z, Chen X. Enhancer of polycomb coordinates multiple signaling pathways to promote both cyst and germline stem cell differentiation in the Drosophila adult testis. PLoS Genet. 2017;13:e1006571.

22. Boivin A, Gally C, Netter S, Anxolabehere D, Ronsseray S. Telomeric associated sequences of Drosophila recruit polycombgroup proteins in vivo and can induce pairing-sensitive repression. Genetics. 2003;164:195-208.

23. White-Cooper H, Schafer MA, Alphey LS, Fuller MT. Transcriptional and post-transcriptional control mechanisms coordinate the onset of spermatid differentiation with meiosis I in Drosophila. Development. 1998;125:125-34.

24. Baker CC, Gim BS, Fuller MT. Cell type-specific translational repression of cyclin B during meiosis in males. Development. 2015;142:3394-402.

25. Jacobs HW, Knoblich JA, Lehner CF. Drosophila cyclin B3 is required for female fertility and is dispensable for mitosis like cyclin B. Genes Dev. 1998;12:3741-51.

26. Soto MC, Chou TB, Bender W. Comparison of germline mosaics of genes in the polycomb group of Drosophila melanogaster. Genetics. 1995; 140:231-43.

27. Boudreault AA, et al. Yeast enhancer of polycomb defines global Esa1-dependent acetylation of chromatin. Genes Dev. 2003; 17:1415-28.

28. Lorbeck M, Pirooznia K, Sarthi J, Zhu X, Elefant F. Microarray analysis uncovers a role for Tip60 in nervous system function and general metabolism. PLoS ONE. 2011;6:e18412.

29. Tea JS, Luo L. The chromatin remodeling factor Bap55 functions through the TIP60 complex to regulate olfactory projection neuron dendrite targeting. Neural Dev. 2011;6:5.

30. Ruthenburg AJ, Allis CD, Wysocka J. Methylation of lysine 4 on histone H3: intricacy of writing and reading a single epigenetic mark. Mol Cell. 2007;25:15-30.

31. Kirmizis A, et al. Silencing of human polycomb target genes is associated with methylation of histone H3 Lys 27. Genes Dev. 2004; 18:1592-605.

32. Kiger AA, Jones DL, Schulz C, Rogers MB, Fuller MT. Stem cell self-renewal specified by JAK-STAT activation in response to a support cell cue. Science. 2001;294:2542-5.

33. Tulina N, Matunis E. Control of stem cell self-renewal in Drosophila spermatogenesis by JAK-STAT signaling. Science. 2001;294:2546-9.

34. Leatherman JL, Dinardo S. Germline self-renewal requires cyst stem cells and stat regulates niche adhesion in Drosophila testes. Nat Cell Biol. 2010;12:806-11.

35. Tarayrah L, Li Y, Gan Q, Chen X. Epigenetic regulator Lid maintains germline stem cells through regulating JAK-STAT signaling pathway activity. Biol Open. 2015;4:1518-27. 
36. Kawase E, Wong MD, Ding BC, Xie T. Gbb/Bmp signaling is essential for maintaining germline stem cells and for repressing bam transcription in the Drosophila testis. Development. 2004;131:1365-75.

37. Inaba M, Yuan H, Salzmann V, Fuller MT, Yamashita YM. E-cadherin is required for centrosome and spindle orientation in Drosophila male germline stem cells. PLoS ONE. 2010;5: e12473.

38. Maggert KA, Golic KG. Highly efficient sex chromosome interchanges produced by I-CreI expression in Drosophila. Genetics. 2005;171:1103-14.

39. Stracker TH, Usui T, Petrini JH. Taking the time to make important decisions: the checkpoint effector kinases Chk1 and Chk2 and the DNA damage response. DNA Repair (Amst). 2009;8:1047-54.

40. Ma X, et al. DNA damage-induced CHK2 activation compromises germline stem cell self-renewal and lineage differentiation. Development. 2016;143:4312-23.

41. Baker CC, Fuller MT. Translational control of meiotic cell cycle progression and spermatid differentiation in male germ cells by a novel eIF4G homolog. Development. 2007;134:2863-9.

42. Monk AC, et al. HOW is required for stem cell maintenance in the Drosophila testis and for the onset of transit-amplifying divisions. Cell Stem Cell. 2010;6:348-60.

43. Voichek Y, Bar-Ziv R, Barkai N. Expression homeostasis during DNA replication. Science. 2016;351:1087-90.

44. Flegel K, Grushko O, Bolin K, Griggs E, Buttitta L. Roles for the histone modifying and exchange complex $\mathrm{NuA} 4$ in cell cycle progression in Drosophila melanogaster. Genetics. 2016;203:1265-81.

45. Monk AC, Siddall NA, Fraser B, McLaughlin EA, Hime GR. Differential roles of HOW in male and female Drosophila germline differentiation. PLoS One. 2011;6:e28508.

46. Ji S, et al. Bam-dependent deubiquitinase complex can disrupt germ-line stem cell maintenance by targeting cyclin A. Proc Natl Acad Sci USA. 2017;114:6316-21.
47. Landais S, D'Alterio C, Jones DL. Persistent replicative stress alters polycomb phenotypes and tissue homeostasis in Drosophila melanogaster. Cell Rep. 2014;7:859-70.

48. Yan D, et al. A regulatory network of Drosophila germline stem cell self-renewal. Dev Cell. 2014;28:459-73.

49. Nakahata $\mathrm{S}$, et al. Alteration of enhancer of polycomb 1 at $10 \mathrm{p} 11.2$ is one of the genetic events leading to development of adult T-cell leukemia/lymphoma. Genes Chromosomes Cancer. 2009;48:768-76.

50. Biankin AV, et al. Pancreatic cancer genomes reveal aberrations in axon guidance pathway genes. Nature. 2012;491:399-405.

51. Rong YS, et al. Targeted mutagenesis by homologous recombination in D. melanogaster. Genes Dev. 2002;16:1568-81.

52. Eun SH, Shi Z, Cui K, Zhao K, Chen X. A non-cell autonomous role of $\mathrm{E}(\mathrm{z})$ to prevent germ cells from turning on a somatic cell marker. Science. 2014;343:1513-6.

53. Lu KL, Yamashita YM. Germ cell connectivity enhances cell death in response to DNA damage in the Drosophila testis. Elife. 2017;6:e27960.

54. Madigan JP, Chotkowski HL, Glaser RL. DNA double-strand break-induced phosphorylation of Drosophila histone variant $\mathrm{H} 2 \mathrm{Av}$ helps prevent radiation-induced apoptosis. Nucleic Acids Res. 2002;30:3698-705.

55. Kumar SV, Wigge PA. H2A.Z-containing nucleosomes mediate the thermosensory response in Arabidopsis. Cell. 2010;140:136-47.

56. Asadzadeh J, Neligan N, Kramer SG, Labrador JP. Tinman regulates NetrinB in the cardioblasts of the Drosophila dorsal vessel. PLoS ONE. 2016;11:e0148526.

57. Bras-Pereira C, et al. Dachshund potentiates Hedgehog signaling during Drosophila retinogenesis. PLoS Genet. 2016;12:e1006204.

58. Castel SE, et al. Dicer promotes transcription termination at sites of replication stress to maintain genome stability. Cell. 2014;159:572-83.

59. Saredi G, et al. H4K20me0 marks post-replicative chromatin and recruits the TONSL-MMS22L DNA repair complex. Nature. 2016;534:714-8. 